\title{
A COMPUTATIONAL APPROACH FOR THE INVERSE PROBLEM OF NEURONAL CONDUCTANCES DETERMINATION
}

\author{
JEMY A. MANDUJANO VALLE, ALEXANDRE L. MADUREIRA, AND ANTONIO LEITÃO
}

\begin{abstract}
The derivation by Alan Hodgkin and Andrew Huxley of their famous neuronal conductance model relied on experimental data gathered using the squid giant axon. However, the experimental determination of conductances of neurons is difficult, in particular under the presence of spatial and temporal heterogeneities, and it is also reasonable to expect variations between species or even between different types of neurons of the same species.

We tackle the inverse problem of determining, given voltage data, conductances with non-uniform distribution in the simpler setting of a passive cable equation, both in a single or branched neurons. To do so, we consider the minimal error iteration, a computational technique used to solve inverse problems. We provide several numerical results showing that the method is able to provide reasonable approximations for the conductances, given enough information on the voltages, even for noisy data.
\end{abstract}

\section{INTRODUCTION.}

The seminal model of Hodgkin and Huxley [23] of neuronal voltage conductance describes how action potential occurs and propagates. It is a landmark model and presents an outstanding combination of modeling based on physical arguments and experimental data, needed to determine the behavior of ion channels. As a part of their work, they modeled the macroscopic behavior of the conductances by designing several mathematical functions (the $\alpha$ 's and $\beta$ 's) that make the computed voltage to behave as the data. In this paper, we propose a numerical procedure to approximate the conductances of the ion channels, using an iterative method to obtain the unknown parameters.

Finding the conductances is crucial if one wants to emulate the neuronal voltage propagation using computational models, since the conductances are part of the data required by the

Date: May 12, 2020.

Key words and phrases. cable equation, conductances determination, inverse problems.

The first author would like to thank PCI-CNPq (301330/2020-4) for its financial suport. Also, the second author acknowledges the support of CNPq (grant 307392/2018-0) and FAPERJ (grant E-26/210.162/2019), and the third author acknowledges support from the research agency CNPq (grant 311087/2017-5), and from the AvH Foundation. 
Hodgkin and Huxley model. Using simpler models might be an alternative, but it is always necessary to find out what are the physiological parameters. What we would like to offer is a computational way to determine the conductances based on experimental data, and we consider our method a step towards that final goal. The method can also be extended to accommodate excitatory and inhibitory synapses, and could be used, in principle, in several nonlinear models, such as the FitzHugh-Nagumo, Morris-Lecar, Hodgkin-Huxley, etc, with varying degrees of difficulty. We remark that our protocol differs from the experimental setup of Hodgkin-Huxley since voltage-clamp techniques are no longer needed, rendering the method simpler and amenable for use even considering heterogeneous spatially distributed channels.

We use a simplified neuronal model, the passive cable equation [4, 20, 44], given by a parabolic partial differential equation (PDE). We consider first the case of a tree with no branches, i.e., possessing only a trunk represented by the interval $[0, L]$. The case of a branched tree is described in Section 2.1. In the cable model the membrane electrical potential $V:[0, T] \times[0, L] \rightarrow \mathbb{R}$ solves

$$
C_{M} V_{t}=\frac{r_{a}}{2 R} V_{x x}-I(t, x) \quad \text { for } \quad 0<t<T, \quad 0<x<L,
$$

where $C_{M}$ represents membrane specific capacitance in microfarad per square centimeter $\left(\mu \mathrm{F} / \mathrm{cm}^{2}\right)$; the potential $V$ is in millivolt $(\mathrm{mV})$; the time $t$ is in milliseconds $(m s) ; r_{a}$ is the radius of the fiber in millicentimeter $(\mathrm{mcm})$; the specific resistance $R$ is in ohm centimeter $(\Omega \mathrm{cm})$; the space $x$ is in centimeter $(\mathrm{cm})$; the specific ionic current $I$ is in microampere per square centimeter $\left(\mu \mathrm{A} / \mathrm{cm}^{2}\right)$. For the passive cable models, the ionic current is given by

$$
I(t, x)=G_{L}\left(V-E_{L}\right)+\sum_{i \in \operatorname{Ion}} G_{i}(t, x)\left(V(t, x)-E_{i}\right),
$$

where the constant leak specific conductance $G_{L}$ is in millisiemens per square centimeter $\left(\mathrm{mS} / \mathrm{cm}^{2}\right) ; E_{L}$ represents leak equilibrium potential in millivolt $(\mathrm{mV})$; Ion is the set of ions of the model, e.g., Ion $=\{\mathrm{K}, \mathrm{Na}\}$. Also, the membrane specific conductance $G_{i}$ for the ion $i \in$ Ion is in millisiemens per square centimeter $\left(\mathrm{mS} / \mathrm{cm}^{2}\right)$, and it might depend on spatial and temporal variables, as indicated in the notation. Finally, the Nernst potential $E_{i}$ for each ion $i \in$ Ion is given in millivolt $(m V)$.

To Eq. (1) we add boundary and initial conditions given by

$$
V_{x}(t, 0)=p(t), \quad V_{x}(t, L)=q(t), \quad V(0, x)=r(x), \text { for } 0<t<T, \quad 0<x<L .
$$

We assume that the constants $C_{M}, r_{a}, R, G_{L}, E_{L}, E_{i}, T$ and $L$, and the functions $p, q$ and $r$ are given data. 
We next rewrite Eqs. (1) and (2) in a slightly more convenient form

$$
\begin{cases}C_{M} V_{t}=\frac{r_{a}}{2 R} V_{x x}-G_{L}\left(V-E_{L}\right)-\sum_{i \in I o n} G_{i}(t, x)\left[V-E_{i}\right], & \text { in }(0, T) \times(0, L), \\ V(0, x)=r(x), & \text { in } x \in[0, L], \\ V_{x}(t, 0)=p(t), \quad V_{x}(t, L)=q(t), & \text { in } t \in[0, T] .\end{cases}
$$

Let $N_{\text {ion }}$ be the number of ions of the set Ion. For Ion $=\left\{1,2, \cdots, N_{\text {ion }}\right\}, \boldsymbol{G}(t, x)=$ $\left(G_{1}(t, x), \ldots, G_{N_{\text {ion }}}(t, x)\right)$. The inverse problem of finding the "correct" $\boldsymbol{G}$ given measurements of the voltage is highly nontrivial, in the sense that it leads to ill-posed problems [50], and that it becomes even harder in the presence of spatially dependent parameters. There are different approaches to deal with the problem in hand, but certainly no panacea.

Hodgkin and Huxley [23] tackled such problem by a highly nontrivial data fitting, in a wonderful achievement made possible only due to an ingenious combination of experimental and biophysical insight. We refer to [6] for a quite interesting description of how experimental results evolved through time. From this same reference we quote:

"The simplicity of the squid axon [...] was rarely found in other cells such as neurons or cardiac myocytes"

and it would be interesting to have a way to combine experimental data and numerical simulation to unveil the macroscopic conductance behavior even in the absence of such simplicity. Wilfrid Rall and co-authors considered several related questions for the cable equation [38, 39, 40, 41, 42, 24]. See also [45, 25, 7, 18, 16, 43, 28]. In [51] there is an interesting attempt to introduce heterogeneity into the Hodgkin and Huxley model.

In terms of biologically inclined references, the authors of [53] consider the branched cable equation with the chemical synapses and convert somatic conductances into dendritic conductances. There are several other articles [3, 30, 48, 49, 52] dealing with the issue of determining conductances and pre-synaptic inputs with different techniques, ranging from deterministic to statistical and stochastic. However, it is far from clear if their approach can be mathematically justified and if it is possible at all to extend those ideas for spatially distributed conductances.

We consider next references with a stronger mathematical flavor. The uniqueness of solutions for finding constant parameters in the cable equation, and related methods, are discussed in [10, 13, 15], and [12] for a nonlinear model; see also [1, 37] for further considerations related to existence and uniqueness. In [14, a related problem was tackled based on the FitzHugh-Nagumo and Morris-Lecar models, where nonlinear functions modeling the conductances are sought. The method is based on fixed point arguments, and despite its 
ingenuity, it is not clear how to extend it to more involved models or to accommodate for spatially distributed ions channels.

In [5, 46, 11, 1, 2], the question of determining spatially distributed conductances is investigated through different techniques and algorithms. They differ considerably from our method and seem harder to generalize for other situations, as, for instance, when the domain is given by branched trees (with the obvious exception of [1, 2]), for time-dependent conductances, and for general nonlinear equations, our ultimate goal.

We would like to stress that although neuroscience models based on ordinary differential equations are, and will always be, of paramount importance, it is our belief that spatially distributed equations will grow in importance. And spatially distributed data will become easier to gather, in particular, due to techniques as voltage-sensitive dye imaging (VSDI) [8, 22 .

Inverse problems like the present one are ill-posed, and, under certain conditions, the minimal error method [21, 26, 36] provides a convergent iterative scheme. The main goal of the present paper is to develop such method to approximate the inverse problem of recovering the conductances in the cable equation. We also test the scheme under different scenarios.

The minimal error method is one of several iterative regularization methods for obtaining stable solutions for ill-posed problems. It has the advantage of each iteration being "cheap" (it avoids inversions present in Newton-like methods) at the possible price of taking more iterations to converge. See [35] for a nice review and comparison among the methods.

We next outline the contents of the paper. In Section 2, we present the method, detailing how it should be applied in the cases of non-branched and branched cables, where the geometry is given by a tree. Section 3 presents numerical results, and in Section 4 we draw some concluding remarks. Finally, the Appendix provides some technical details regarding the method and the mathematics behind it.

\section{Method: The minimal ERror scheme Applied to the COnductance DETERMINATION}

We consider here an application of the minimal error method to the problem at hand. Knowing the voltage $V$ at the time-space domain $\Gamma$, we want to determine $\boldsymbol{G}$ assuming that Eq. (3) holds. We consider two different cases in voltage measurement. In the first case, we assume that $V$ is known at all time-space points, i.e., $\Gamma=[0, T] \times[0, L]$ (Table 1 , Case I). In the second case, we assume that the voltage is known only at endpoints and all the time. Thus $\Gamma=[0, T] \times\{0, L\}($ Table 1 , Case II). 


$$
\begin{array}{|l|l|}
\hline \text { CASE I } & \Gamma=[0, T] \times[0, L]=\{(t, x) ; \quad 0 \leq t \leq T, \quad 0 \leq x \leq L\} \\
\hline \text { CASE II } & \Gamma=[0, T] \times\{0, L\}=\{(t, x) ; \quad 0 \leq t \leq T, \quad x \in\{0, L\}\} \\
\hline
\end{array}
$$

TABle 1. Summary of the two different cases considered in this paper. We seek the unknowns $G_{i}$ assuming that Eq. (3) holds and that a measure of the voltage $V$ is known at the space-time domain $\Gamma$ defined above. In case $\mathrm{I}$, the data is known at all points and at all times; in case II, the data is known at two end-points and at all times.

Let $\left.V\right|_{\Gamma}$ be the restriction of $V$ to $\Gamma$, and consider the nonlinear operator

$$
F: D(F) \rightarrow R(F)
$$

that associates for a given $\boldsymbol{G} \in D(F)$ the resulting voltage, i.e., $F(\boldsymbol{G})=\left.V\right|_{\Gamma}$, where $V$ solves Eq. (3). The domain $D(F)$ and the image $R(F)=L^{2}(\Gamma)$ (the space of square integrable functions) are properly defined in the Appendix A. Given a smooth enough function $f$, we define its $L^{2}$ norm $\|\cdot\|_{L^{2}(\Gamma)}$ such that

$$
\|f\|_{L^{2}(\Gamma)}^{2}=\int_{\Gamma}|f(\xi)|^{2} d \xi
$$

We consider the inverse problem of finding an approximation for $\boldsymbol{G}$ given the noisy data $\left.V^{\delta}\right|_{\Gamma}$, where

$$
\left\|V-V^{\delta}\right\|_{L^{2}(\Gamma)} \leq \delta
$$

for some known noise threshold $\delta>0$. That makes sense since, in practice, the data $\left.V\right|_{\Gamma}$ are never known exactly. In Section 3 we detail the type of noise introduced.

Given an initial guess $\boldsymbol{G}^{1, \delta}$, the minimal error approximation for $\boldsymbol{G}$ is defined by the sequence

$$
\boldsymbol{G}^{k+1, \delta}=\boldsymbol{G}^{k, \delta}+w^{k, \delta} F^{\prime}\left(\boldsymbol{G}^{k, \delta}\right)^{*}\left(\left.V^{\delta}\right|_{\Gamma}-F\left(\boldsymbol{G}^{k, \delta}\right)\right)
$$

for $k=1,2, \ldots$, where $F^{\prime}(\cdot)^{*}$ is adjoint of the Gâteux derivative, and

$$
w^{k, \delta}=\frac{\left\|V^{\delta}-F\left(\boldsymbol{G}^{k, \delta}\right)\right\|_{L^{2}(\Gamma)}^{2}}{\left\|F^{\prime}\left(\boldsymbol{G}^{k, \delta}\right)^{*}\left(V^{\delta}-F\left(\boldsymbol{G}^{k, \delta}\right)\right)\right\|_{D(F)}^{2}} .
$$

The minimal error iteration is a gradient method, as is the steepest descent method. Although the steps of both methods follow the same direction (the gradient), they differ on 
their step size [26]. As a stopping criteria we use the discrepancy principle with $\tau>1$, i.e., we define the stopping iteration step $k_{*}$ such that

$$
\left\|V^{\delta}-F\left(\boldsymbol{G}^{k_{*}, \delta}\right)\right\|_{L^{2}(\Gamma)} \leq \tau \delta \leq\left\|V^{\delta}-F\left(\boldsymbol{G}^{k, \delta}\right)\right\|_{L^{2}(\Gamma)}
$$

for all $1 \leq k<k_{*}$. In practice, stopping criteria are needed for all iterative methods, otherwise the scheme might stop before it is accurate enough, or diverge, or even waste computing time without significantly improving the solution. For inverse problems with noisy data this is even more crucial since running regularization iterative methods beyond certain threshold forces the method to "fit the noise". It is possible to show that, under certain conditions (we assume that is the case), $\boldsymbol{G}^{k_{*}, \delta}$ converges to a solution of $F(\boldsymbol{G})=V$ as $\delta \rightarrow 0$; see [26, Theorem 2.6].

From Eqs. (6) and (8) we obtain an approximation $\boldsymbol{G}^{k_{*}, \delta}$ for $\boldsymbol{G}$. Although the adjoint $F^{\prime}\left(\boldsymbol{G}^{k, \delta}\right)^{*}$ is not known, it is possible to show that Eq. (6) is actually

$$
G_{i}^{k+1, \delta}(t, x)=G_{i}^{k, \delta}(t, x)-w^{k, \delta}\left(V^{k, \delta}(t, x)-E_{i}\right) U^{k}(t, x) \quad \text { for all } i \in \text { Ion, }
$$

where

$$
w^{k, \delta}=\frac{\left\|V^{\delta}-F\left(\boldsymbol{G}^{k, \delta}\right)\right\|_{L^{2}(\Gamma)}^{2}}{\sum_{i \in \text { Ion }}\left\|\left(V^{k, \delta}(t, x)-E_{i}\right) U^{k}(t, x)\right\|_{D(F)}^{2}} .
$$

Also $V^{k, \delta}$ solves Eq. (3) with $\boldsymbol{G}$ replaced by $\boldsymbol{G}^{k, \delta}$, and $U^{k}$ solves the following PDE with final condition:

$$
\begin{cases}-\frac{r_{a}}{2 R} U_{x x}-C_{M} U_{t}+G_{L} U+\sum_{i \in \operatorname{Ion}} G_{i}(t, x) U=\alpha_{1}\left(V^{\delta}-V\right), & \text { in }(0, T) \times(0, L), \\ U(T, x)=0, & \text { in } x \in[0, L], \\ U_{x}(t, 0)=-\alpha_{2} \frac{2 R}{r_{a}}\left(V^{\delta}(t, 0)-V(t, 0)\right), & \text { in } t \in[0, T], \\ U_{x}(t, L)=\alpha_{2} \frac{2 R}{r_{a}}\left(V^{\delta}(t, L)-V(t, L)\right), & \text { in } t \in[0, T] .\end{cases}
$$

The constants $\alpha_{1}, \alpha_{2}$ depend on the set $\Gamma$ as follows:

$$
\left(\alpha_{1}, \alpha_{2}\right)= \begin{cases}(1,0) & \text { if } \Gamma=[0, T] \times[0, L], \\ (0,1) & \text { if } \Gamma=[0, T] \times\{0, L\} .\end{cases}
$$

In Theorem A.1 of the Appendix, we show how to obtain Eq. (9) from Eq. (6).

Remark 1. Note from Eq. (9) that $G_{i}^{k+1, \delta}(T, x)=G_{i}^{k, \delta}(T, x)$ for all $x \in[0, L]$ and every $k \in \mathbb{N}$, since, from Eq. (10), $U^{k}(T, x)=0$. Thus, $G_{i}^{k, \delta}$ is never corrected at the final time $T$. 
To recover $G_{i}$ at time $T$, one could perform the computations up to $2 T$, and then consider only the values up to $T$.

The numerical scheme of our method is as follows. Check Table 1 for notation. Note from Algorithm 1 that solutions of two PDEs are needed for each iteration.

Data: $\left.V^{\delta}\right|_{\Gamma}, r, p, q, \delta, \tau$

Result: Compute an approximation for $\boldsymbol{G}$ using minimal error Iteration Scheme

Choose $\boldsymbol{G}^{1, \delta}$ as an initial approximation for $\boldsymbol{G}$;

Compute $V^{1, \delta}$ from Eq. (3), replacing $\boldsymbol{G}$ by $\boldsymbol{G}^{1, \delta}$;

$\mathrm{k}=1$;

while $\tau \delta \leq\left\|V^{\delta}-V^{k, \delta}\right\|_{L^{2}(\Gamma)}$ do

Compute $U^{k}$ from Eq. (10);

Compute $\boldsymbol{G}^{k+1, \delta}$ using Eq. (9);

Compute $V^{k+1, \delta}$ from Eq. (3), replacing $\boldsymbol{G}$ by $\boldsymbol{G}^{k+1, \delta}$;

$k \leftarrow k+1$;

end

Algorithm 1: Minimal Error Iteration

Remark 2. Whenever $\boldsymbol{G}$ is time independent, and in this case we write $\boldsymbol{G}(t, x)=\boldsymbol{G}(x)$, the iteration is defined by

$$
G_{i}^{k+1, \delta}(x)=G_{i}^{k, \delta}(x)-w^{k, \delta} \frac{1}{T} \int_{0}^{T}\left(V^{k, \delta}(t, x)-E_{i}\right) U^{k}(t, x) d t \quad \text { for } i \in \text { Ion },
$$

where

$$
w^{k, \delta}=\frac{\left\|V^{\delta}-F\left(\boldsymbol{G}^{k, \delta}\right)\right\|_{L^{2}(\Gamma)}^{2}}{\sum_{i \in \text { Ion }}\left\|\frac{1}{T} \int_{0}^{T}\left(V^{k, \delta}(t, x)-E_{i}\right) U^{k}(t, x) d t\right\|_{L^{\infty}(0, L)}^{2}} .
$$

\subsection{The minimal error method applied to the conductance determination defined}

on a tree. We now describe the equivalent formulation when the domain is given by a tree, and we consider for simplicity a tree with a single branch. Note however that the generalizations for more complex geometries are straightforward. In general, the tree $\Theta=$ $\mathcal{E} \cup \mathcal{V}$ is defined by the set of edges $\mathcal{E}$ and the set of vertices $\mathcal{V}$. Boundary conditions are imposed on the terminal vertices $\mathcal{V}_{T}$, and transmission conditions are imposed on the interior vertices $\mathcal{V}_{I}$. Of course, $\mathcal{V}_{T} \cup \mathcal{V}_{I}=\mathcal{V}$. In the case depicted in Figure 1 .

$$
\mathcal{E}=\left\{e_{1}, e_{2}, e_{3}\right\}, \quad \mathcal{V}=\left\{\nu_{1}, \nu_{2}, \nu_{3}, \nu_{4}\right\}, \quad \mathcal{V}_{T}=\left\{\nu_{1}, \nu_{3}, \nu_{4}\right\}=\left\{\gamma_{1}, \gamma_{2}, \gamma_{3}\right\}, \quad \mathcal{V}_{I}=\left\{\nu_{2}\right\}
$$




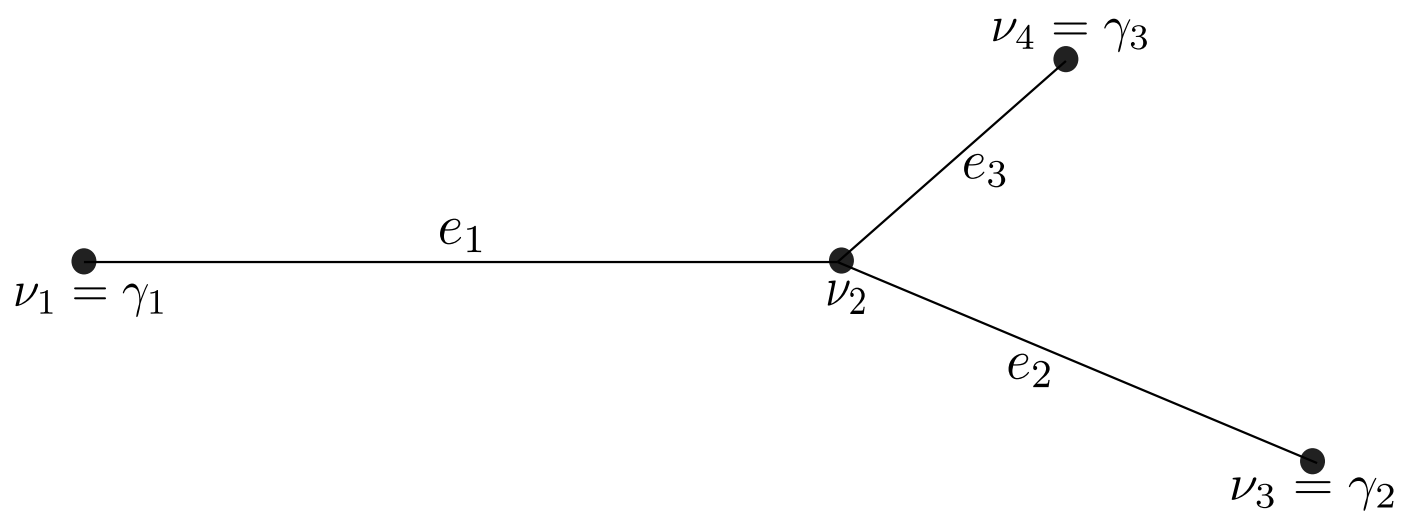

FIGURE 1. Example of a branched tree with one bifurcation point.

Our cable equation model defined on a branched tree is given by

$$
\begin{cases}C_{M} V_{t}=\frac{r_{a}}{2 R} V_{x x}-G_{L}\left(V-E_{L}\right)-\sum_{i \in \text { Ion }} G_{i}(t, x)\left[V-E_{i}\right], & \text { in }(0, T) \times \mathcal{E}, \\ V(0, x)=r(x), & \text { in } x \in \Theta, \\ V_{x}\left(t, \gamma_{1}\right)=p(t), & \text { in } t \in[0, T], \\ V_{x}\left(t, \gamma_{2}\right)=V_{x}\left(t, \gamma_{3}\right)=q(t), & \text { in } t \in[0, T], \\ V_{x}^{e_{1}}\left(t, \nu_{2}\right)-V_{x}^{e_{2}}\left(t, \nu_{2}\right)-V_{x}^{e_{3}}\left(t, \nu_{2}\right)=0, & \text { in } t \in[0, T],\end{cases}
$$

where $V_{x}^{e_{j}}\left(t, \nu_{2}\right)$ denotes the derivative of $V$ at the vertex $\nu_{2}$ taken along the edge $e_{j} \in$ $\left\{e_{1}, e_{2}, e_{3}\right\}$ in the direction towards the vertex.

Consider the operator (4), where $F(\boldsymbol{G})=V(\cdot, \cdot)$ and $V$ solves Eq. (13). Given $V^{\delta}$, our goal is to obtain an approximation to $\boldsymbol{G}$ using the iteration (6). To compute the adjoint operator $F^{\prime}(\cdot)^{*}$, we define the following PDE:

$$
\begin{cases}-\frac{r_{a}}{2 R} U_{x x}^{k}-C_{M} U_{t}^{k}+G_{L} U^{k}+\sum_{i \in \text { Ion }} G_{i}^{k, \delta}(t, x) U^{k}=V^{\delta}-V^{k, \delta}, & \text { in }(0, T) \times \mathcal{E}, \\ U^{k}(T, x)=0, & \text { in } x \in \Theta, \\ U_{x}^{k}(t, 0)=U_{x}^{k}\left(t, \nu_{2}\right)=U_{x}^{k}\left(t, \nu_{3}\right)=0, & \text { in } t \in[0, T], \\ U_{x}^{k^{e_{1}}}\left(t, \nu_{1}\right)-U_{x}^{k^{e_{2}}}\left(t, \nu_{1}\right)-U_{x}^{e^{e_{3}}}\left(t, \nu_{1}\right)=0, & \text { in } t \in[0, T] .\end{cases}
$$

We then compute $G_{i}^{k+1, \delta}$ according to (9). Remarks $1 / 2$ also hold for this problem.

\section{Results: Numerical Simulation}

In this section we test the method under different scenarios. Of course, the solutions are obtained numerically, and for that we use finite difference scheme in space coupled with 
backward Euler in time. To compute the integral in Eq. 12 we use the trapezoidal rule. In what follows we assume that the numerical approximations are accurate enough. All the experiments were performed using Matlab $^{\circledR}$, and the codes are available at [32].

To design our in silico experiments, we first choose $\boldsymbol{G}$ and compute $V$ from Eq. (3), obtaining then $\left.V\right|_{\Gamma}$. Of course, in practice, only the values of $\left.V^{\delta}\right|_{\Gamma}$ are given by some experimental measures, and thus subject to experimental/measurement errors. In our examples, $\left.V^{\delta}\right|_{\Gamma}$ is obtained by considering additive-multiplicative noise

$$
V^{\delta}(t, x)=V(t, x)+(a V+b) \operatorname{rand}_{\Delta}(t, x) \text { for all }(t, x) \in \Gamma,
$$

for scalars $a, b$, and rand $\Delta$ is a uniformly distributed random variable taking values in the range $[-\Delta, \Delta]$. The threshold $\delta$ is such that (cf. Eq. (5)) $\left\|(a V+b) \operatorname{rand}_{\Delta}\right\|_{L^{2}(\Gamma)} \leq \delta$, and we impose then

$$
\|(a V+b)\|_{L^{2}(\Gamma)} \Delta=\delta .
$$

In our numerical examples, we use multiplicative and additive noises, i.e., $a=1 / 2$ and $b=1 / 2$ at Eq. (15). We noticed no qualitative difference in the results for other values of $a$ and $b$.

Remark 3. It is not possible in general to predict how the added noise will affect the conductances since the operator $F$ defined in Eq. (4) is not bounded, meaning that small perturbation of the data might lead to large perturbation of the conductances. That is why inverse problems are so hard to approximate.

Next, given the initial guess $\boldsymbol{G}^{1, \delta}$, the data $\left.V^{\delta}\right|_{\Gamma}$, and the noise threshold $\delta$, we approximate $\boldsymbol{G}$ using the Algorithm 1. Unlike in "direct" PDE problems where the exact solution usually has to be computed by numerical over-kill, here we have the exact $\boldsymbol{G}$ and we use that to gauge the algorithm performance.

In our numerical examples we consider $M=50$ experiments for each $\delta$. Therefore, for an experiment $j$, we measured voltage $\left.V^{\delta}\right|_{\Gamma j}$ and compute the approximation $\boldsymbol{G}_{j}^{k_{*}, \delta}$ of unknown conductance $\boldsymbol{G}$. We define the mean and standard deviation for a function $f$ (in what follows, $f$ will be either $\left.V^{\delta}\right|_{\Gamma}$ or $\left.\boldsymbol{G}^{k_{*}, \delta}\right)$ :

$$
\mu_{f}(t, x)=\frac{1}{M} \sum_{j=1}^{M} f_{j}(t, x), \quad \sigma_{f}(t, x)=\sqrt{\frac{1}{M} \sum_{j=1}^{M}\left(f_{j}(t, x)-\mu_{f}(t, x)\right)^{2}},
$$

if $f=G^{k_{*}, \delta}$ and Ion $=\left\{1,2, \cdots, N_{\text {ion }}\right\}$ then $\mu_{f}=\left(\mu_{G_{1}^{k_{*}, \delta}}, \mu_{G_{2}^{k_{*}, \delta}}, \cdots, \mu_{G_{\mathbb{N}_{\text {ion }}}^{k_{*}, \delta}}\right)$. 
From (8), for iteration $k^{*}$, we introduce

$$
\text { Error }_{\boldsymbol{G}}=\frac{1}{N_{\text {ion }}} \sum_{i \in I o n} \int_{D(\boldsymbol{G})} \frac{\left|G_{i}-\mu_{G_{i}^{k_{*}, \delta}}\right|}{\left|G_{i}\right|} \times 100 \%
$$

where $D(\boldsymbol{G})$ denotes the domain where $\boldsymbol{G}$ is defined. Given value $V$ and its approximation $\mu_{\left.V^{\delta}\right|_{\Gamma}}$, we define the error

$$
\operatorname{Error}_{V}=\int_{\Gamma} \frac{\left|V(t, x)-\mu_{\left.V^{\delta}\right|_{\Gamma}}(t, x)\right|}{|V(t, x)|} d \Gamma \times 100 \% .
$$

Remark 4. There seems to be no common agreement in the literature on what would be the "best" error measurement, and the choice of the above Mean Absolute Percentage error is fully justified in, e.g., [17]. In practice, after discretizing the equations and the unknown functions, only nodal values are known. Consider the space-time discretization $t_{n}=(n-$ 1) $T /(N-1)$ for $n=1,2 \cdots, N$ and $x_{j}=(j-1) L /(J-1)$ for $j=1,2 \cdots, J$. Thus, the relative error introduced above (18) is approximated by

$$
\text { Error }_{\boldsymbol{G}}=\frac{1}{N_{\text {ion }}} \frac{T}{N} \frac{L}{J} \sum_{i \in \text { Ion }} \sum_{n=1}^{J} \sum_{j=1}^{N}\left|\frac{G_{i}\left(t_{n}, x_{j}\right)-G_{i}^{k, \delta}\left(t_{n}, x_{j}\right)}{G_{i}\left(t_{n}, x_{j}\right)}\right| \times 100 \% .
$$

Whenever $\boldsymbol{G}$ is time independent, and in this case we write $\boldsymbol{G}\left(t_{n}, x_{j}\right)=\boldsymbol{G}\left(x_{j}\right)$, the mean absolute percentage error is defined by

$$
\operatorname{Error}_{\boldsymbol{G}}=\frac{1}{N_{\text {ion }}} \frac{L}{J} \sum_{i \in \operatorname{Ion}} \sum_{n=1}^{J}\left|\frac{G_{i}\left(x_{j}\right)-G_{i}^{k, \delta}\left(x_{j}\right)}{G_{i}\left(x_{j}\right)}\right| \times 100 \% .
$$

Similar remark holds for other norms, e.g., for $\Gamma=[0, T] \times[0, L],\|f\|_{L^{2}(\Gamma)}$ is to be replaced by $\|f\|_{l^{2}(\Gamma)}$, where

$$
\|f\|_{l^{2}(\Gamma)}^{2}=\frac{T}{N} \frac{L}{J} \sum_{\left(t_{n}, x_{j}\right) \in \Gamma}\left|f\left(t_{n}, x_{j}\right)\right|^{2}
$$

Remark 5. The mean absolute percentage error, for (19) and $\Gamma=[0, T] \times[0, L]$, is

$$
\text { Error }_{V}=\frac{T}{N} \frac{L}{J} \sum_{n=1}^{J} \sum_{j=1}^{N}\left|\frac{V\left(t_{n}, x_{j}\right)-\mu_{\left.V^{\delta}\right|_{\Gamma}}\left(t_{n}, x_{j}\right)}{V\left(t_{n}, x_{j}\right)}\right| \times 100 \%
$$

and, for (19) and $\Gamma=[0, T] \times\{0, L\}$, is

$$
\text { Error }_{V}=\frac{1}{2} \frac{T}{N} \sum_{j=1}^{N}\left[\left|\frac{V\left(t_{n}, 1\right)-\mu_{\left.V^{\delta}\right|_{\Gamma}}\left(t_{n}, 1\right)}{V\left(t_{n}, 1\right)}\right|+\left|\frac{V\left(t_{n}, J\right)-\mu_{\left.V^{\delta}\right|_{\Gamma}}\left(t_{n}, J\right)}{V\left(t_{n}, J\right)}\right|\right] \times 100 \%
$$


We present four numerical tests. In the first three examples the geometry is defined by a segment, and in the fourth example it is given by a branched tree. The first example considers only one ion (Ion $=\{\mathrm{K}\}$ ), with $\boldsymbol{G}(x)=G_{\mathrm{K}}(x)$ depending only on the spatial variable, and the voltage is known at $\Gamma=[0, T] \times\{0, L\}$, i.e., at all times but only at the endpoints. In the second example, still with one ion (Ion $=\{K\})$, the conductance depends on the temporal and spatial variables $(t, x)$ and measured voltage is known at $\Gamma=[0, T] \times[0, L]$, i.e., all the time and at all points. In the third example, we consider two ions (Ion $=\{\mathrm{K}, \mathrm{Na}\}$ ), where $\boldsymbol{G}(x)=\left(G_{\mathrm{K}}(x), G_{\mathrm{Na}}(x)\right)$ depends only on the spatial variable and the data is again known at $\Gamma=[0, T] \times[0, L]$, i.e., all the time for all points. Finally, in the fourth example we consider the case where the geometry is defined by a tree, with the conductance being time independent under the presence of one ion, and the voltage data being known at all the time and all the points.

For all numerical examples let $C_{M}=1\left(\mu \mathrm{F} / \mathrm{cm}^{2}\right), r_{a}=0.0238(\mathrm{~cm}), R=34.5(\Omega \mathrm{cm})$, $G_{L}=0.3\left(\mathrm{mS} / \mathrm{cm}^{2}\right)$ and $E_{L}=10.613(\mathrm{mV})$; see [47, page 69], [27, page 4] and [9, page 586]. The following Neumann boundary conditions

$$
V_{x}(t, 0)=p(t)=-\frac{R I(t)}{\pi r_{a}{ }^{2}}, \quad V_{x}(t, L)=q(t)=0,
$$

correspond to injecting a current $I(t)$ at $x=0$, and imposing a sealed end condition at $x=L$; see [11, 12, 33, 34]. We consider the current $I(t)=0.1 t^{2} \exp (-10 t)(m A)$ and time $T=20(m s)$, see [12, page 101]. We assume that initial condition $V(0, x)=r(x)=0(m V)$, see [11, 12]. As previously mentioned, we consider $M=50$ experiment for each additivemultiplicative noise (15), where we set $\Delta=25 \%, 5 \%, 1 \%$ and $0.2 \%$ [5, 11, 46]. For stopping criterion (8), we choose $\tau=1.01$. Finally, we set the initial guesses to be zero in the iterative scheme, and thus the initial relative error is $100 \%$.

In Examples 3.1, 3.2 and 3.3, we consider $L=0.1(\mathrm{~cm})$, see [11, page 145]. We solve equations (3) and (10) via finite differences in space, with $\Delta x=0.001(\mathrm{~cm})$, and in time, with $\Delta t=0.2(\mathrm{~ms})$.

For Example 3.4, the length of the edges are: $\left|e_{1}\right|=\left|e_{2}\right|=0.1(\mathrm{~cm})$ and $\left|e_{3}\right|=0.2(\mathrm{~cm})$. The values of the other parameters are the same as above. In this example, we solve the differential equations (13) and (14) again using finite differences, but with explicit Euler in time, and $\Delta x=\Delta t=0.01$.

Example 3.1. Consider a particular instance from Eq. (3), where $N_{i o n}=1($ Ion $=\{K\})$, $E_{K}=-12(m V)$ and $G_{i}(t, x)=G_{K}(x)$. The goal is to estimate

$$
G_{K}(x)=0.2+0.2 /(1+\exp ((0.1 / 2-x) / 0.01))\left(m S / \mathrm{cm}^{2}\right)
$$


given $\left.V^{\delta}\right|_{\Gamma}=\left\{\left(V^{\delta}(t, 0), V^{\delta}(t, L)\right): t \in[0,20]\right\}$. See [11].

In Table 2 we present the results for various levels of noise. At each line of the table the noise is reduced by a factor of five, and that leads to a similar reduction of the residual. The same cannot be stated about the approximation error, exposing the instability of the problem.

In Figures 2 and 3, we plot results for $\Delta=1 \%$ of noise with $M=50$ experiments (see Table 2, line 4). In Figure 2, we display the exact membrane potential, the mean and standard deviation of the voltage measurement (see Eq. (17), for $f=\left.V^{\delta}\right|_{\Gamma}$ ), and the difference between the exact membrane potential and mean of its measurements. In Figure 3, we show the exact conductance, the mean and standard deviation of the approximate solutions (see equation (17), for $f=G^{k_{*}, \delta}$ ), and the difference between the conductance and mean of the approximate solutions. We also consider the higher noise level $\Delta=5 \%$ and present the related figures in the Online Resource.

\begin{tabular}{|l|l|l|}
\hline$\Delta$ & Error $_{G}$ & Error $_{V}$ \\
\hline $25 \%$ & $2.0387 \%$ & $26.4003 \%$ \\
\hline $5 \%$ & $0.7738 \%$ & $4.7587 \%$ \\
\hline $1 \%$ & $0.3306 \%$ & $0.9567 \%$ \\
\hline $0.2 \%$ & $0.2034 \%$ & $0.1893 \%$ \\
\hline
\end{tabular}

TABLE 2. Numerical results for Example 3.1 with $M=50$ experiments for each noise level $\Delta$. The first column describes the noise level $\Delta$, as in Eq. 15. The second column contains the mean errors according to Eq. 21). Finally, the third column contains the mean errors according to Eq. 24).

Example 3.2. We consider conductance as depending on time and space, where $N_{\text {ion }}=1$ $($ Ion $=\{K\}), E_{K}=-12(m V), G_{i}(t, x)=G_{K}(t, x)$. The goal is to estimate

$$
G_{K}(t, x)=0.2+0.2 /(1+\exp ((0.1 / 2-x) / 0.01))+t+1\left(m S / c m^{2}\right)
$$

given $\left.V^{\delta}\right|_{\Gamma}=\left\{V^{\delta}(t, x):(t, x) \in[0,20] \times[0,0.1]\right\}$.

This example is harder than the previous one since now the conductance depends on both time and space. In Table 3 we present the results for various levels of noise, and the same comments of Example 3.1 apply. In Figures 4 and 5 , we plot numerical results for $\Delta=1 \%$ of noise with $M=50$ experiments (see Table 3, line 4). Observe that the data for both $\left.V^{\delta}\right|_{\Gamma}$ and $G_{K}$ depend on time and space. See also our Online Resource for experiments with $\Delta=5 \%$. 

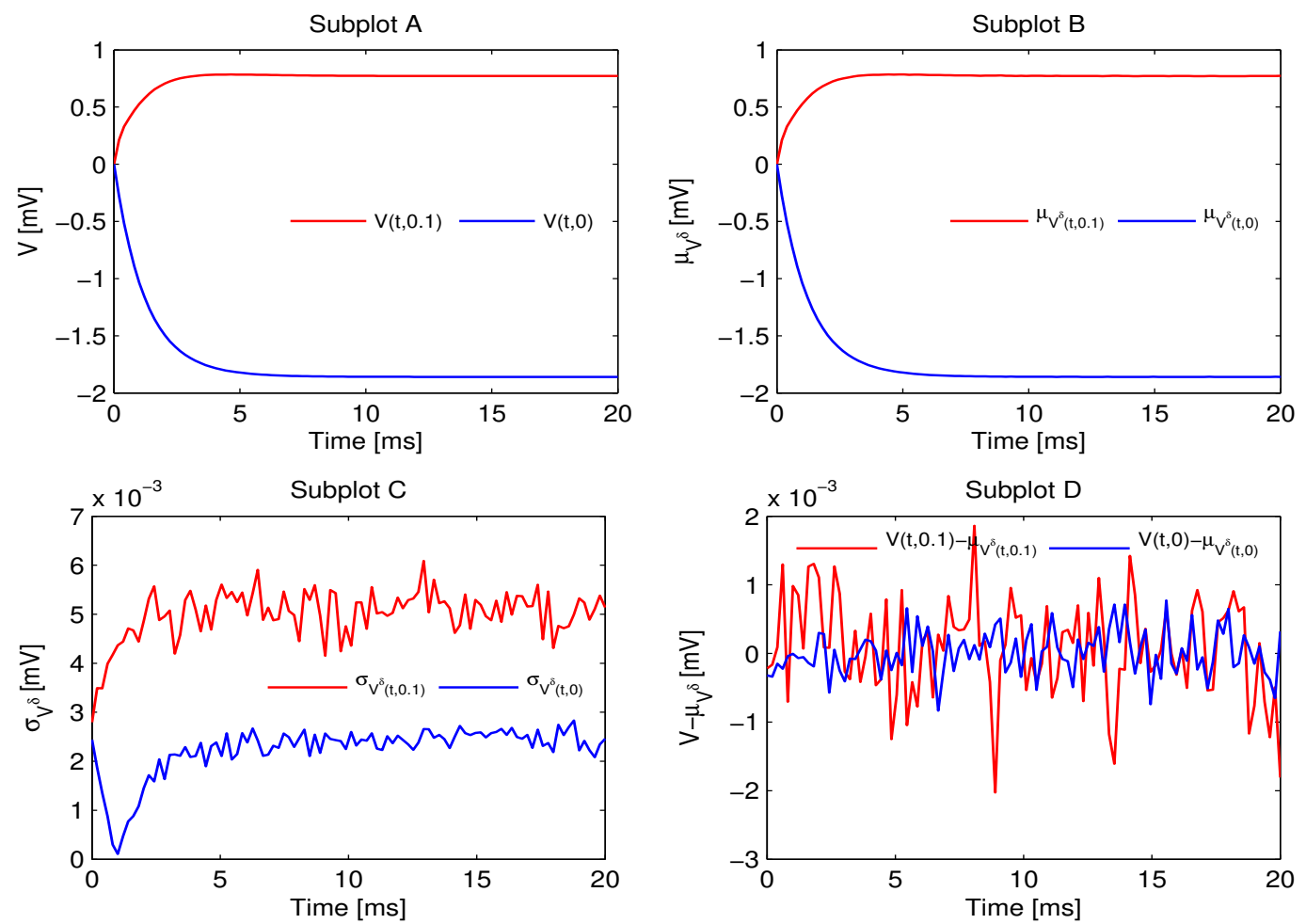

FigurE 2. Result for Example 3.1 with $\Delta=1 \%$ noise. Subplot A presents the exact, deterministic, membrane potential at the end-points. Subplots B and C show the mean and standard deviation of the one hundred membrane potential measurements, respectively. In Subplot D displays the difference between the exact membrane potential and the mean of its measurements.

\begin{tabular}{|l|l|l|}
\hline$\Delta$ & Error $_{G}$ & Error $_{V}$ \\
\hline $25 \%$ & $17.7328 \%$ & $4.2053 \%$ \\
\hline $5 \%$ & $2.3030 \%$ & $0.6823 \%$ \\
\hline $1 \%$ & $0.5655 \%$ & $0.1312 \%$ \\
\hline $0.2 \%$ & $0.2061 \%$ & $0.0309 \%$ \\
\hline
\end{tabular}

TABLE 3. Numerical results for Example 3.2 with $M=50$ experiments for each noise level $\Delta$. The first column describes the noise level $\Delta$, as in Eq. 15). The second column contains the mean errors according to Eq. 20). Finally, the third column contains the mean errors according to Eq. (23).

Example 3.3. Consider now two different ions, $K$ and $N a$, where $N_{\text {ion }}=2($ Ion $=\{K, N a\})$ $E_{K}=-12[\mathrm{mV}]$ and $E_{N a}=115[\mathrm{mV}]$. The goal is to approximate

$$
G_{K}(x)=0.2+0.2 /(1+\exp ((0.1 / 2-x) / 0.01))
$$



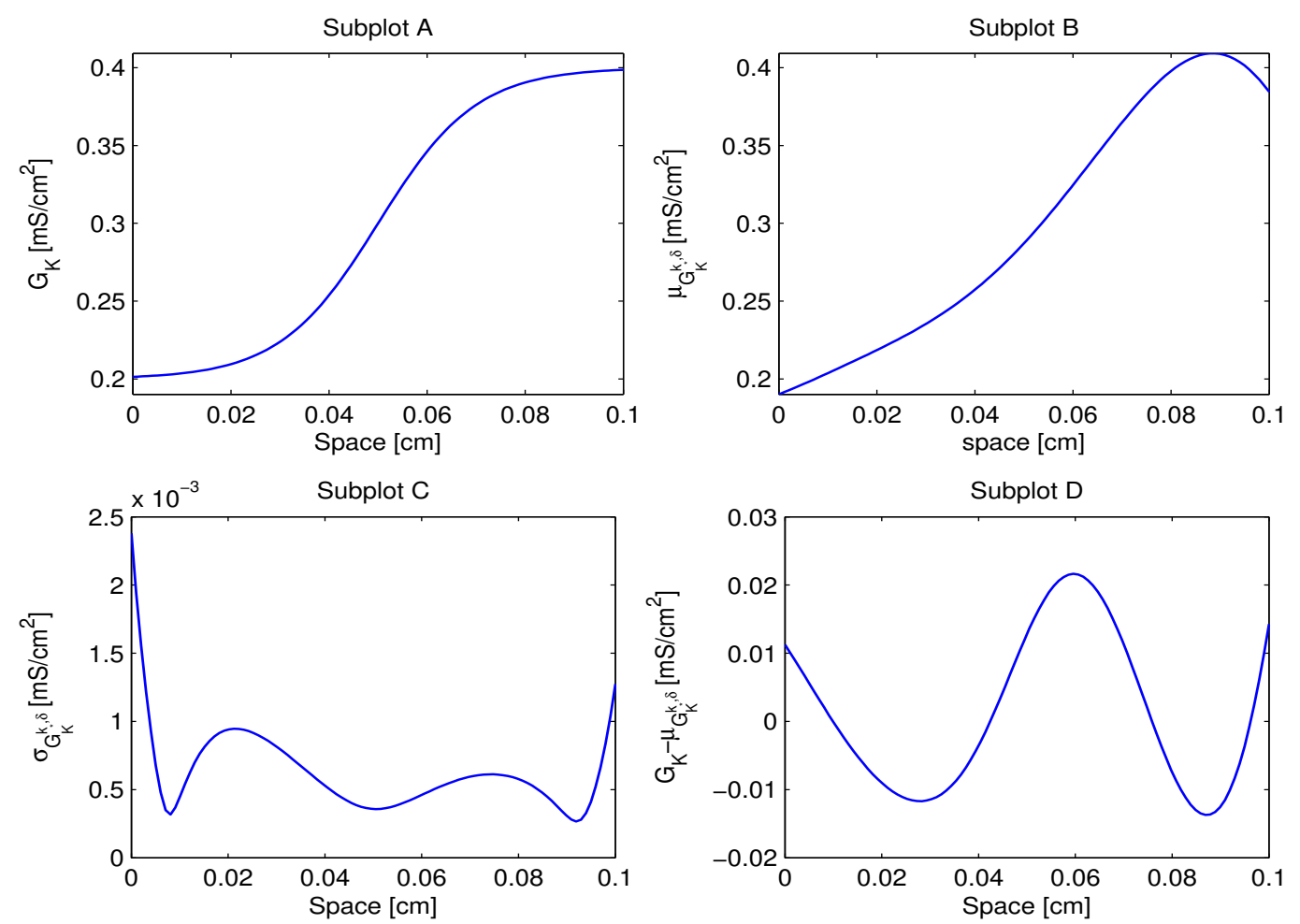

FiguRE 3. For Example 3.1 with $\Delta=1 \%$. Subplot A shows the exact solution $G_{\mathrm{K}}$. In subplots B and C present the mean and standard deviation of the one hundred approximate solutions, respectively. Subplot D displays the difference between the exact solution and mean of the approximate solutions.

and

$$
G_{N a}(x)=0.1+0.1 /(1+\exp ((0.1 / 2-x) / 0.01))
$$

given $\left.V^{\delta}\right|_{\Gamma}=\left\{V^{\delta}(t, x):(t, x) \in[0,20] \times[0,0.1]\right\}$.

The extra difficulty in this example lies in the fact that there are two conductance functions to be discovered. In Table 4 we present the results for various levels of noise. In Figures 6, 9 and 8, we plot results for $\Delta=1 \%$ of noise with $M=50$ experiments (see Table 3, line 3). Note that now there are two conductances, one related to $K$ and the other to $N a$. For results with $\Delta=5 \%$ see the Online Resource.

Example 3.4. As our final example, we consider the domain defined by a tree, as discussed in Section 2.1. We consider $N_{\text {ion }}=1($ Ion $=\{K\}), E_{K}=-12(m V)$ and $G_{i}(t, x)=G_{K}(x)$. Given $V^{\delta}$ in $(0, T) \times \Theta$, the goal is to estimate

$$
G_{K}(x)= \begin{cases}0.2+0.2 /\left(1+\exp \left(\left(0.1 / 2-\operatorname{dist}\left(x, \nu_{1}\right)\right) / 0.01\right)\right) & \text { if } x \in e_{1}, \\ 0.2+0.2 /\left(1+\exp \left(\left(0.1 / 2-0.01-\operatorname{dist}\left(x, \nu_{2}\right)\right) / 0.01\right)\right) & \text { if } x \in e_{2} \cup e_{3},\end{cases}
$$


Subplot A
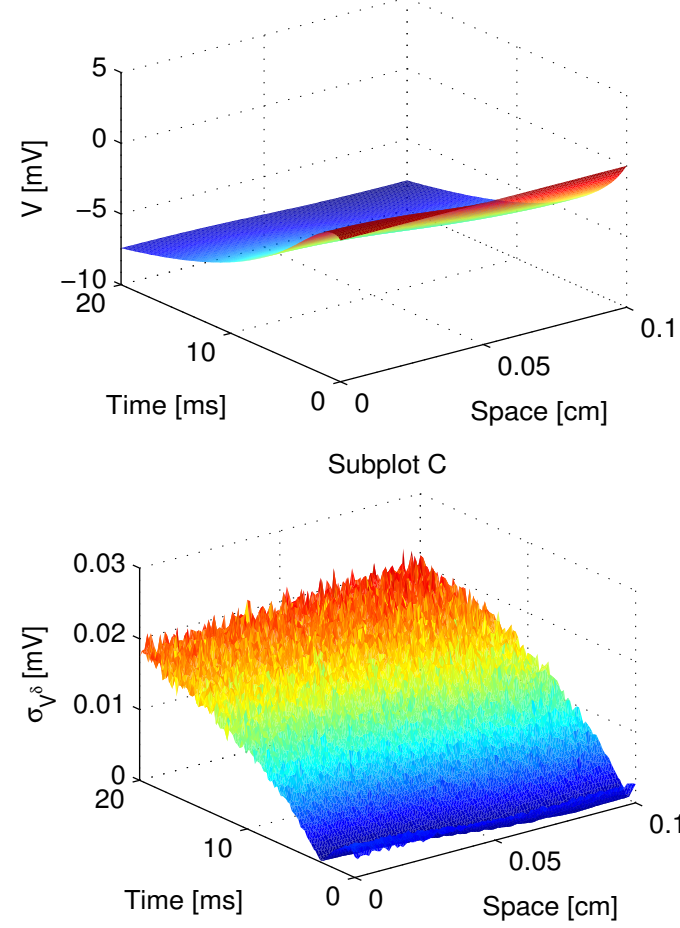

Subplot B

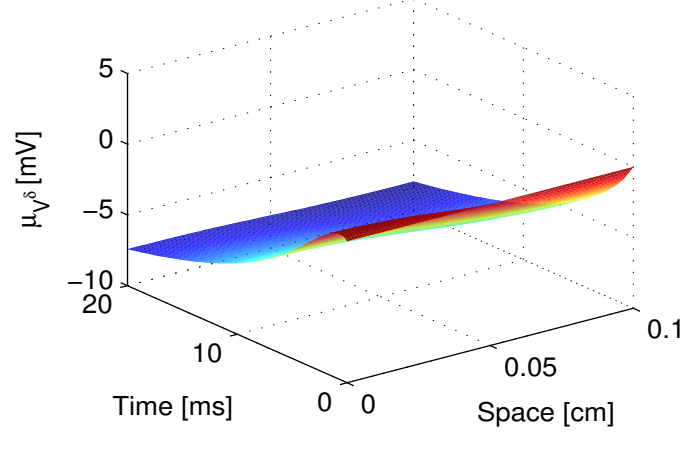

Subplot D

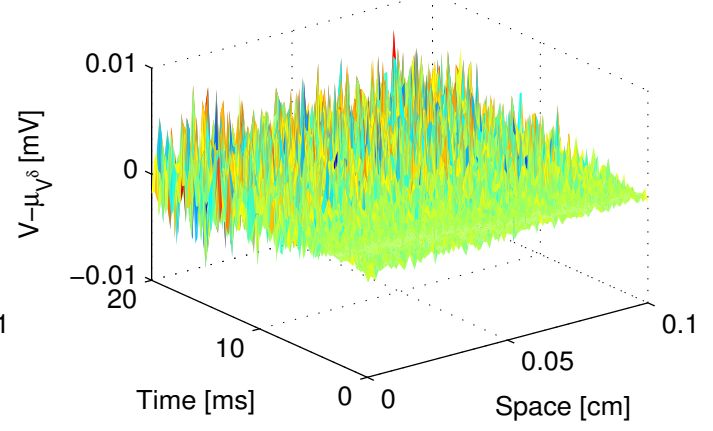

Figure 4. For Example 3.2 with $\Delta=1 \%$. See Figure 2 for the subplots description.

\begin{tabular}{|l|l|l|}
\hline$\Delta$ & Error $_{G}$ & Error $_{V}$ \\
\hline $25 \%$ & $8.2571 \%$ & $1.7057 \%$ \\
\hline $5 \%$ & $2.1458 \%$ & $2.1458 \%$ \\
\hline $1 \%$ & $0.5653 \%$ & $0.0687 \%$ \\
\hline $0.2 \%$ & $0.2109 \%$ & $0.0136 \%$ \\
\hline
\end{tabular}

TABLE 4. Numerical results for Example 3.3 with $M=50$ experiments for each noise level $\Delta$. The first column describes the noise level $\Delta$, as in Eq. 15). The second column contains the mean errors according to Eq. 21). Finally, the third column contains the mean errors according to Eq. 23).

where $\operatorname{dist}(a, b)$ denotes the distance between the points $a$ and $b$. Table 5 presents the results for various levels of noise. In figures 9 14, we plot the numerical result for $\Delta=1 \%$, and in the Online Resource we plot the results for $\Delta=5 \%$.

\section{Conclusions}

The inverse problem of finding conductances from voltage data for a given neuron model is important and difficult. This paper presents and tests a way to approximate them based on 

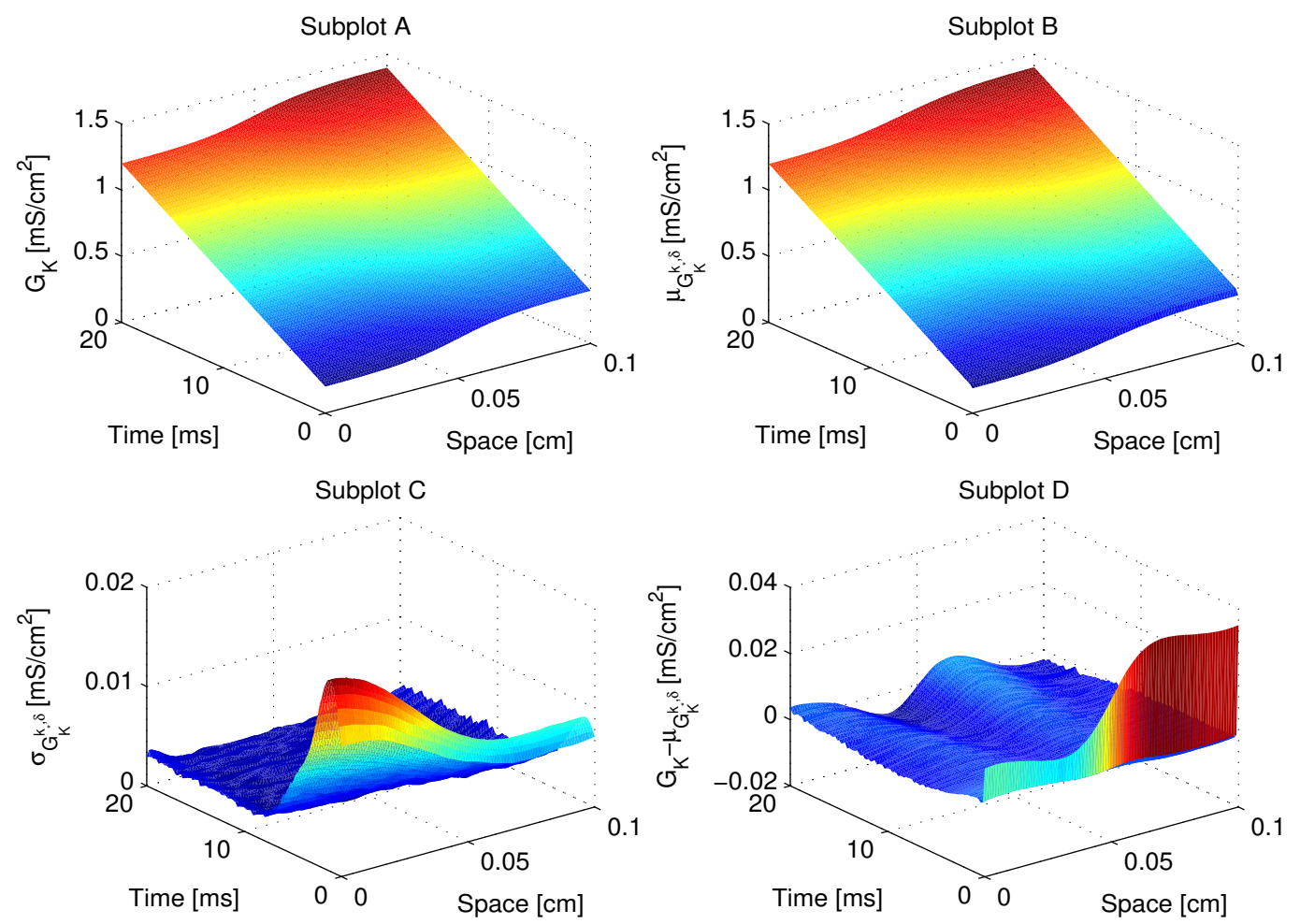

Figure 5. Plots for Example 3.2. See Figure 3 for the subplots description.

\begin{tabular}{|l|l|l|}
\hline$\Delta$ & Error $_{G}$ & Error $_{V}$ \\
\hline $25 \%$ & $2.8572 \%$ & $3.9757 \%$ \\
\hline $5 \%$ & $0.7661 \%$ & $0.8127 \%$ \\
\hline $1 \%$ & $0.2926 \%$ & $0.1605 \%$ \\
\hline $0.2 \%$ & $0.1204 \%$ & $0.0319 \%$ \\
\hline
\end{tabular}

TABLE 5. Numerical results for Example 3.4 with $M=50$ experiments for each noise level $\Delta$. The first column describes the noise level $\Delta$, as in Eq. 15). The second column contains the mean errors according to Eq. (21). Finally, the third column contains the mean errors according to Eq. 23.

the minimal error iterative method and applied to a cable model. Although the scheme has a somewhat straightforward description, it is not practical in its original formulation since computing the adjoint of the Gâteux derivative seems unfeasible in general. The development of auxiliary equations to overcome such hurdle requires some art, and is done on a case-bycase basis.

Certainly, the method has limitations and is no panacea. How well the method performs depends on the noise, on how close to the solution is the initial guess, the amount of data, 

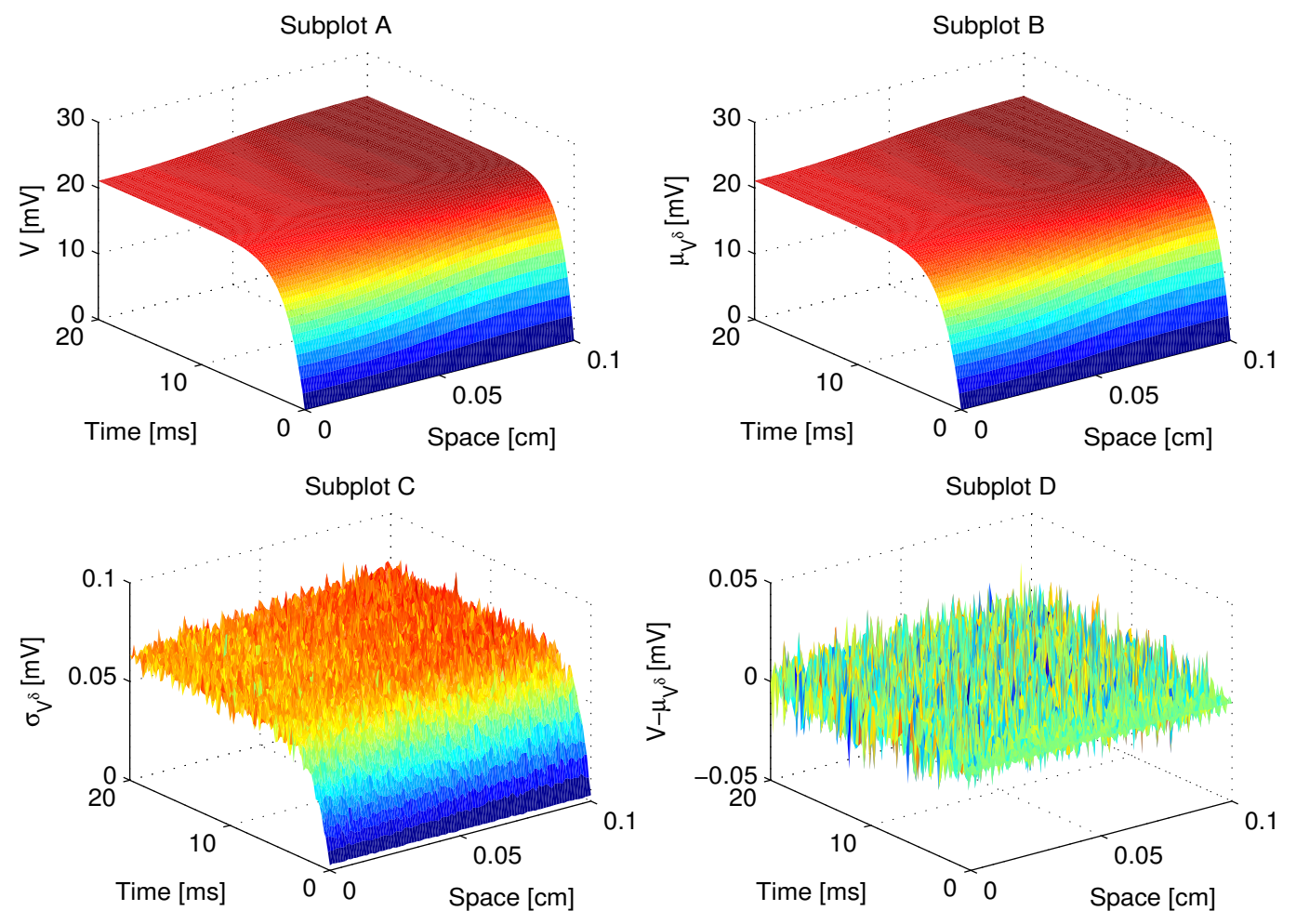

Figure 6. For Example 3.3 with $\Delta=1 \%$. See Figure 2 for the subplots description.

and on the model used. There is a nontrivial interplay between all those conditions. For instance, determining two conductances is harder than determining one, finding conductances that depend on time and space is harder than finding conductances that depend on space only. Also, having data at all points is better than if the data is available at isolated points only.

Our examples display some of these features. For some of them, the method performs nicely, capturing the correct conductances. If the level of noise increases, the method delivers reasonable approximations (see our Online Resource for that), but these approximations cannot be qualitatively better than the available data, especially for inverse problems. Inverse problems are unstable, and thus, not well-posed and difficult to solve in general. Even when the method does not do a good job in capturing the correct conductance, the computed residual is small, and whenever the residual is of the same order as the noise, there is no point in iterating any further.

Under reasonable conditions, the method yields good results even in the presence of noise, as shown here. It is also general enough to accommodate for different geometries (straight cables and branched trees), and different measured data (endpoints, whole cable). 

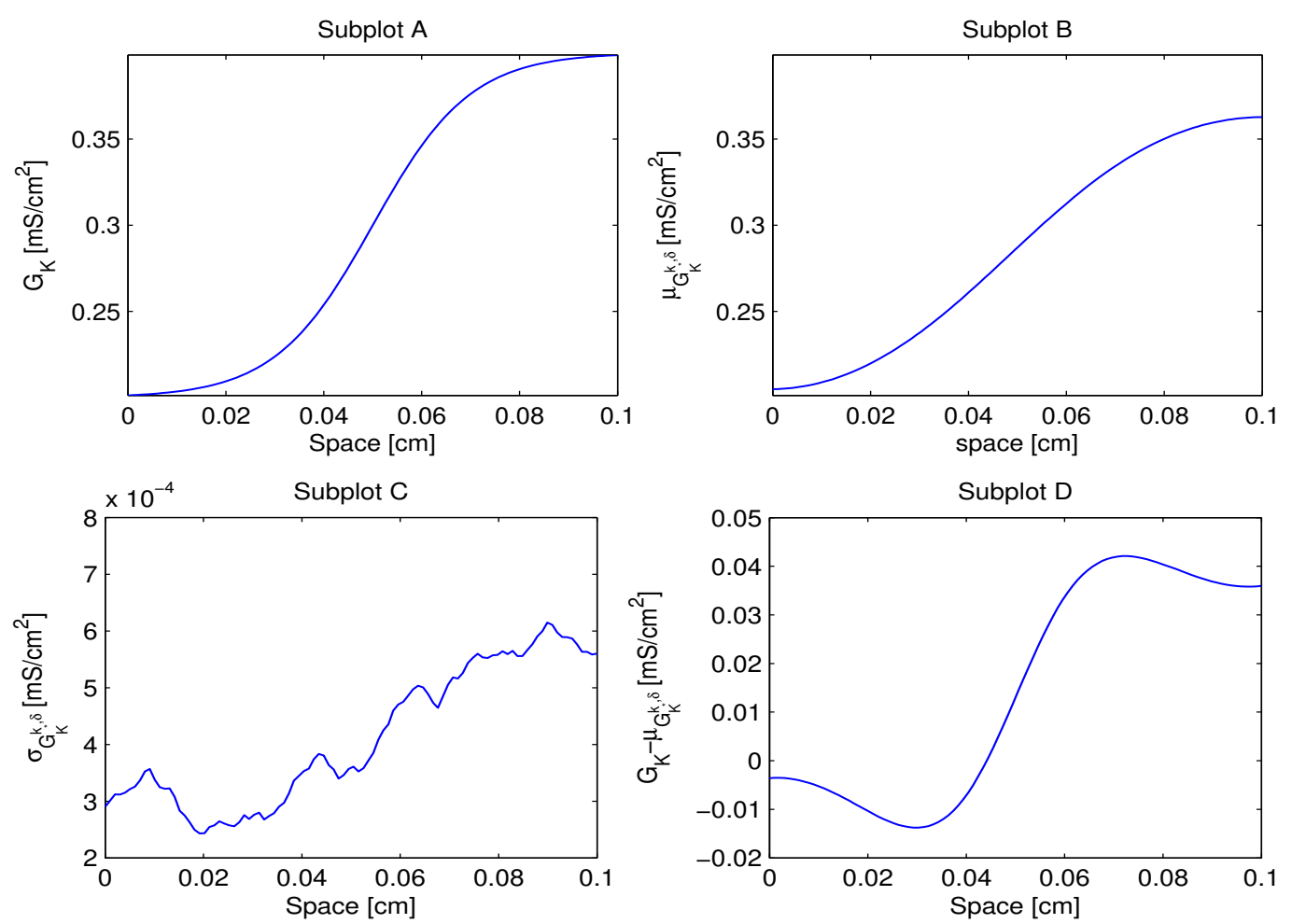

FiguRE 7. Results for Example 3.3 with $\Delta=1 \%$. See Figure 3 for the subplots description..

We believe that methods that are capable of inferring spatial properties of neurons are in demand and will grow in importance, in particular due to new imagining techniques such as VSDI. Also, regularizing methods for inverse problems are applied in several research fields and they can also contribute to Neuroscience.

Finally, we note that inverse problems are nonlinear by nature, even if the equations involved are linear. If the equations are nonlinear, then the problem becomes much harder since finding the adjoint of the Gateaux derivatives of the operators involved is highly nontrivial. The present work represents a first step towards finding conductances for neuronal models. The final goal is to develop a similar method for the Hodgkin-Huxley system, but the highly nonlinear nature of those equations makes the task daunting, even when considering ODEs.

\section{Appendix A. Abstract Formulation}

In practice, $\left.V\right|_{\Gamma}$ is the data and given such information and under the assumption that Eq. (3) holds, the inverse problem under consideration is to recover or approximate the conductances. The lack of stability, characteristic of ill-posed problems can be tamed by regularization methods [19, 26, 29], in particular by the minimal error method. 

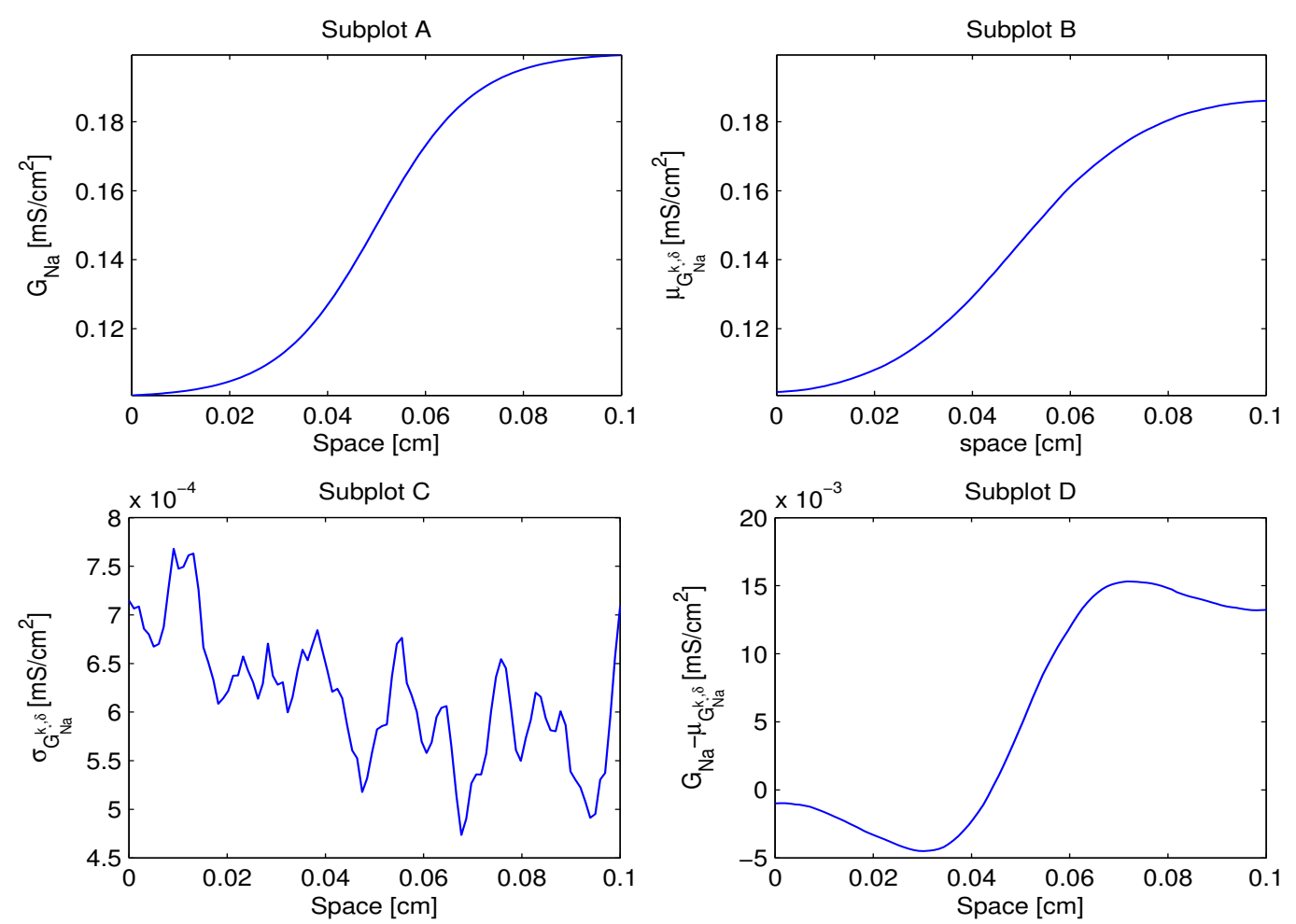

FiguRE 8. Results for Example 3.3 with $\Delta=1 \%$ of noise. See Figure 3 for the subplots description.

Consider for simplicity $T>0$. Let $\Omega=\{(t, x): 0 \leq t \leq T, 0 \leq x \leq L\}$, and

$$
\begin{gathered}
H(F)=\left(L^{2}(\Omega)\right)^{N_{\text {ion }}}=\left\{f: \Omega \rightarrow \mathbb{R}^{N_{\text {ion }}}: \int_{\Omega}|f(\xi)|^{2} d \xi<\infty\right\}, \\
R(F)=L^{2}(\Gamma)=\left\{f: \Gamma \rightarrow \mathbb{R}: \int_{\Gamma}|f(\xi)|^{2} d \xi<\infty\right\} .
\end{gathered}
$$

It is well-known that $H(F)$ and $R(F)$ become Hilbert spaces under the inner products

$$
\langle f, h\rangle_{H(F)}=\int_{\Omega} f(\xi) h(\xi) d \xi, \quad\langle f, h\rangle_{R(F)}=\int_{\Gamma} f(\xi) h(\xi) d \xi, .
$$

and the associated norms $\|f\|_{H(F)}=\langle f, f\rangle_{H(F)}^{1 / 2},\|f\|_{R(F)}=\langle f, f\rangle_{R(F)}^{1 / 2}$. Note that the inner product on $R(F)$ depends on $\Gamma$, see $E q$. (11), as follows:

$$
\begin{aligned}
\langle f, h\rangle_{R(F)}=\alpha_{1} \int_{0}^{L} \int_{0}^{T} f(t, x) h(t, x) d t d x+\alpha_{2} \int_{0}^{T} f(t, 0) h(t, 0) d t & \\
& +\alpha_{2} \int_{0}^{T} f(t, L) g(t, L) d t
\end{aligned}
$$

where $\alpha_{1}, \alpha_{2}$ are as in Eq. (11). 


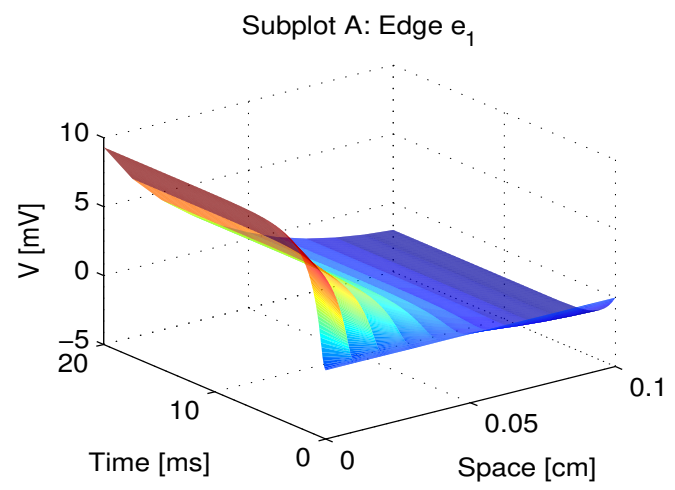

Subplot C: Edge $e_{1}$

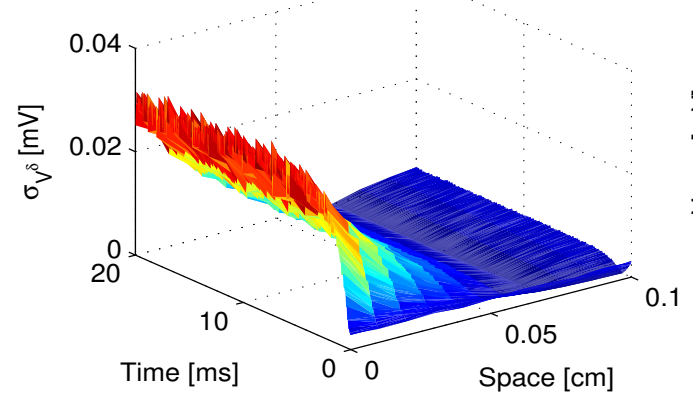

Subplot B: Edge $\mathrm{e}_{1}$

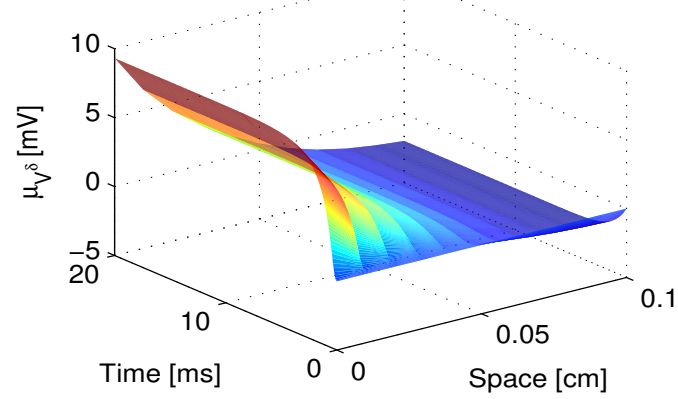

Subplot D: Edge $e_{1}$

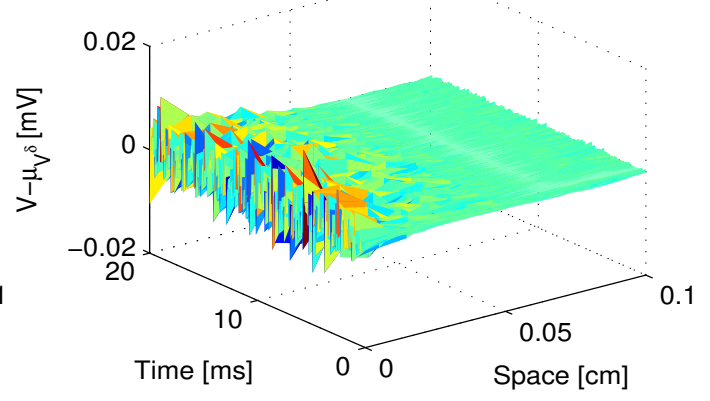

FigurE 9. For Example 3.4 and edge $e_{1}$, with $\Delta=1 \%$. See Figure 2 for the subplots description.

The set $D(F)=\left(L^{\infty}(\Omega)\right)^{N_{\text {ion }}} \subset H(F)$ is the Banach space of "essentially" bounded functions (see [31] for precise definitions). Consider the operator $F: D(F) \subset H(F) \rightarrow R(F)$ defined by $F(\boldsymbol{G})=\left.V\right|_{\Gamma}$. Our goal is to find an approximation for $\boldsymbol{G}$ using the minimal error iteration defined by Eq. (6).

In the next Theorem we show how to obtain Eq. (9) from Eq. (6).

Theorem A.1. Consider the iteration in Eq. (6). Then Eq. (9) holds

Proof. Given $\boldsymbol{G}^{k, \delta} \in D(F)$ and $\boldsymbol{\theta}=\left(\theta_{1}, \ldots, \theta_{N_{\text {ion }}}\right) \in H(F)$, the Gâteux derivative of $F$ at $\boldsymbol{G}^{k, \delta}$ in the direction $\boldsymbol{\theta}$ is given by

$$
F^{\prime}\left(\boldsymbol{G}^{k, \delta}\right)(\boldsymbol{\theta})=\lim _{\lambda \rightarrow 0} \frac{F\left(\boldsymbol{G}^{k, \delta}+\lambda \boldsymbol{\theta}\right)-F\left(\boldsymbol{G}^{k, \delta}\right)}{\lambda}=\left.W^{k}\right|_{\Gamma},
$$



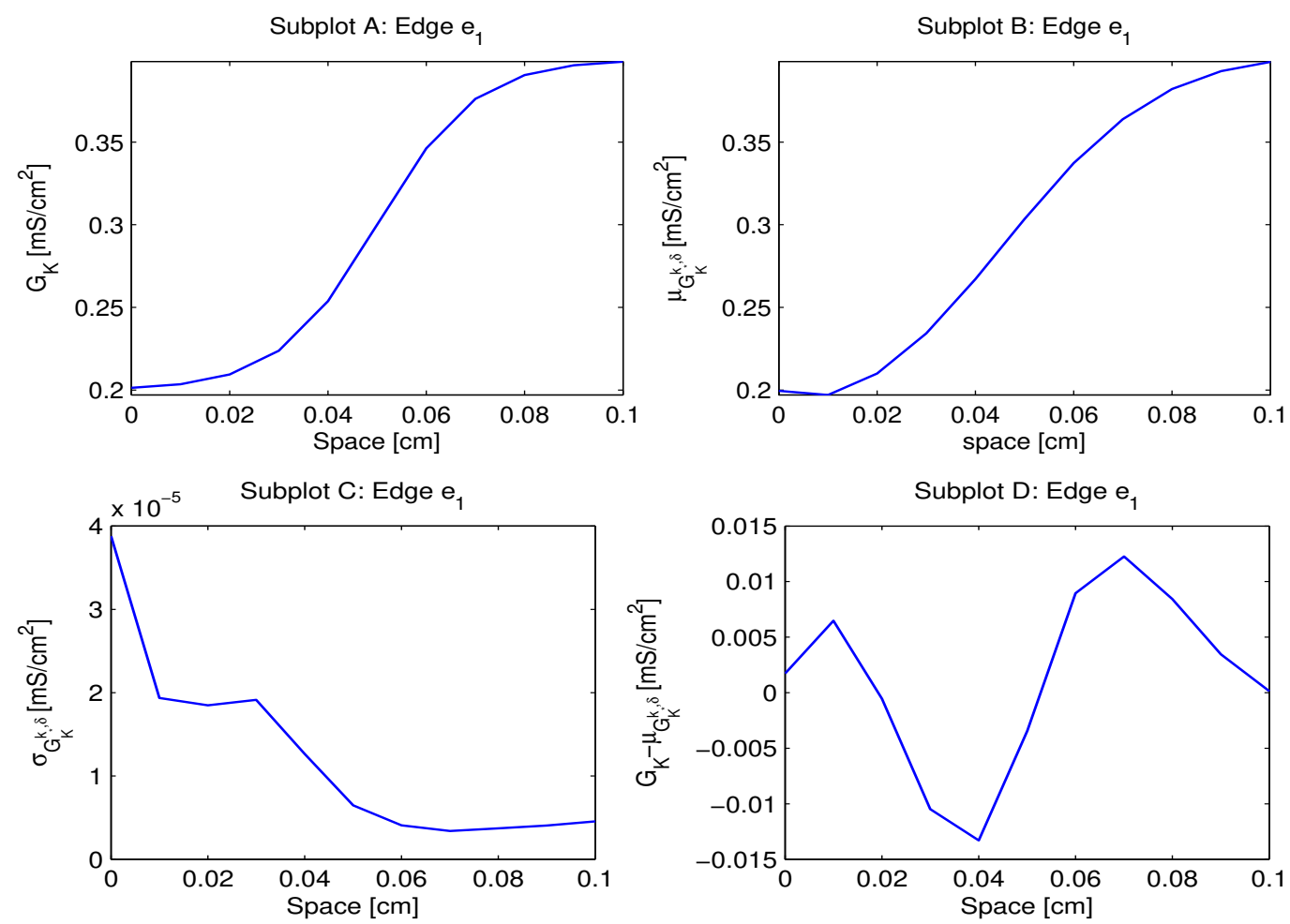

Figure 10. Plots for Example 3.4 and edge $e_{1}$. See Figure 3 for the subplots description.

where $W^{k}$ solves

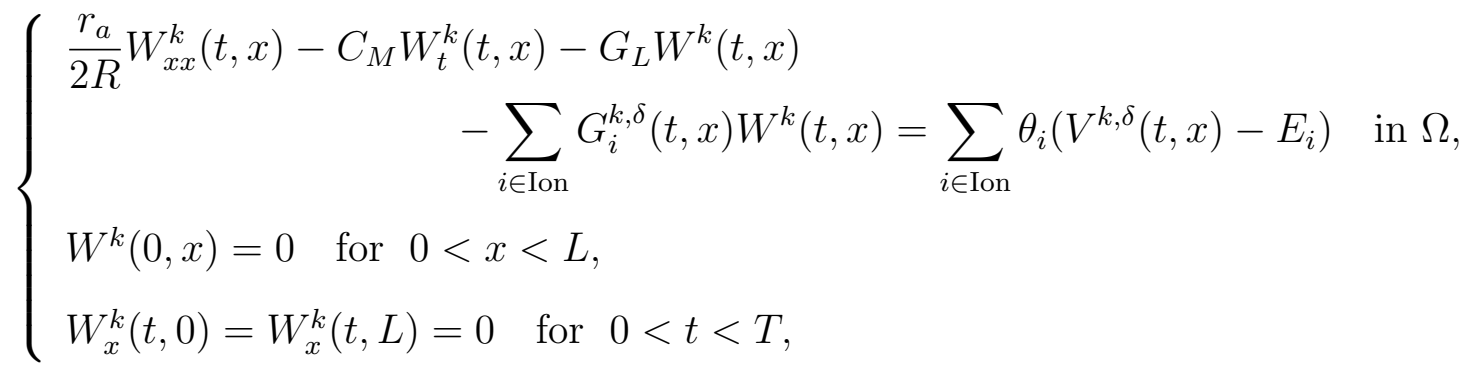

and $V^{k, \delta}$ solves Eq. (3) with $G_{i}$ replaced by $G_{i}^{k, \delta}$. To obtain Eq. (27) from Eq. (26), it is enough to consider the difference between problem in Eq. (3) with coefficients $\boldsymbol{G}^{k, \delta}+\lambda \boldsymbol{\theta}$ and $\boldsymbol{G}^{k, \delta}$, divide by $\lambda$ and take the limit $\lambda \rightarrow 0$.

Let $\left.V^{k, \delta}\right|_{\Gamma}=F\left(\boldsymbol{G}^{k, \delta}\right)$. From the minimal error iteration in Eq. (6), we gather that

$$
\begin{aligned}
\left\langle\boldsymbol{G}^{k+1, \delta}-\boldsymbol{G}^{k, \delta}, \boldsymbol{\theta}\right\rangle_{H(F)} & =w^{k, \delta}\left\langle F^{\prime}\left(\boldsymbol{G}^{k, \delta}\right)^{*}\left(\left.V^{\delta}\right|_{\Gamma}-F\left(\boldsymbol{G}^{k, \delta}\right)\right), \boldsymbol{\theta}\right\rangle_{H(F)} \\
& =w^{k, \delta}\left\langle F^{\prime}\left(\boldsymbol{G}^{k, \delta}\right)^{*}\left(\left.V^{\delta}\right|_{\Gamma}-\left.V^{k, \delta}\right|_{\Gamma}\right), \boldsymbol{\theta}\right\rangle_{H(F)}
\end{aligned}
$$



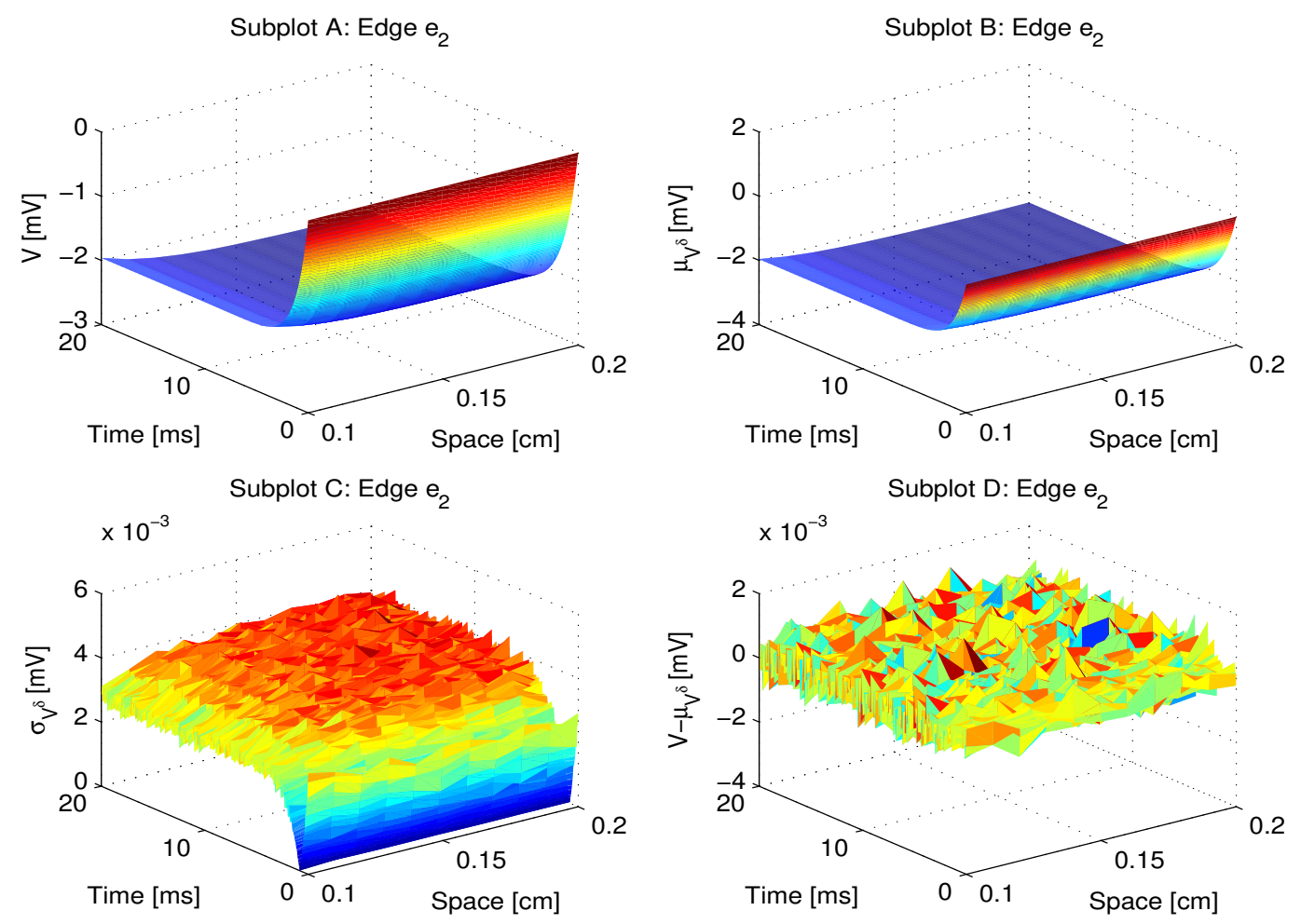

Figure 11. For Example 3.4 and edge $e_{2}$, with $\Delta=1 \%$. See Figure 2 for the subplots description.

By definition of adjoint operator,

$$
\begin{aligned}
\left\langle\boldsymbol{G}^{k+1, \delta}-\boldsymbol{G}^{k, \delta}, \boldsymbol{\theta}\right\rangle_{H(F)}=w^{k, \delta}\left\langle\left. V^{\delta}\right|_{\Gamma}-\left.V^{k, \delta}\right|_{\Gamma}, F^{\prime}\left(\boldsymbol{G}^{k, \delta}\right)(\boldsymbol{\theta})\right\rangle_{R(F)} & \\
& =w^{k, \delta}\left\langle\left. V^{\delta}\right|_{\Gamma}-\left.V^{k, \delta}\right|_{\Gamma},\left.W^{k}\right|_{\Gamma}\right\rangle_{R(F)}
\end{aligned}
$$

from Eq. (26).

Although Eq. (28) yields an interesting relation, it carries an impeding dependence on $\boldsymbol{\theta}$ through $W^{k}$. It is possible to avoid that by performing some "trick" manipulations.

Multiplying the first equation from $(10)$ by $-W^{k}$, and integrating in the intervals $[0, T]$ and $[0, L]$ we gather that

$$
\begin{aligned}
\int_{0}^{L} \int_{0}^{T} \frac{r_{a}}{2 R} U_{x x}^{k}(t, x) W^{k}(t, x) d t d x & +\int_{0}^{L} \int_{0}^{T} C_{M} U_{t}^{k}(t, x) W^{k}(t, x) d t d x \\
-\int_{0}^{L} \int_{0}^{T} G_{L} U^{k}(t, x) W^{k}(t, x) d t d x & -\int_{0}^{L} \int_{0}^{T} \sum_{i \in \text { ion }} G_{i}^{k, \delta}(t, x) U^{k}(t, x) W^{k}(t, x) d t d x= \\
& -\alpha_{1} \int_{0}^{L} \int_{0}^{T}\left(V^{\delta}(t, x)-V^{k, \delta}(t, x)\right) W^{k}(t, x) d t d x .
\end{aligned}
$$



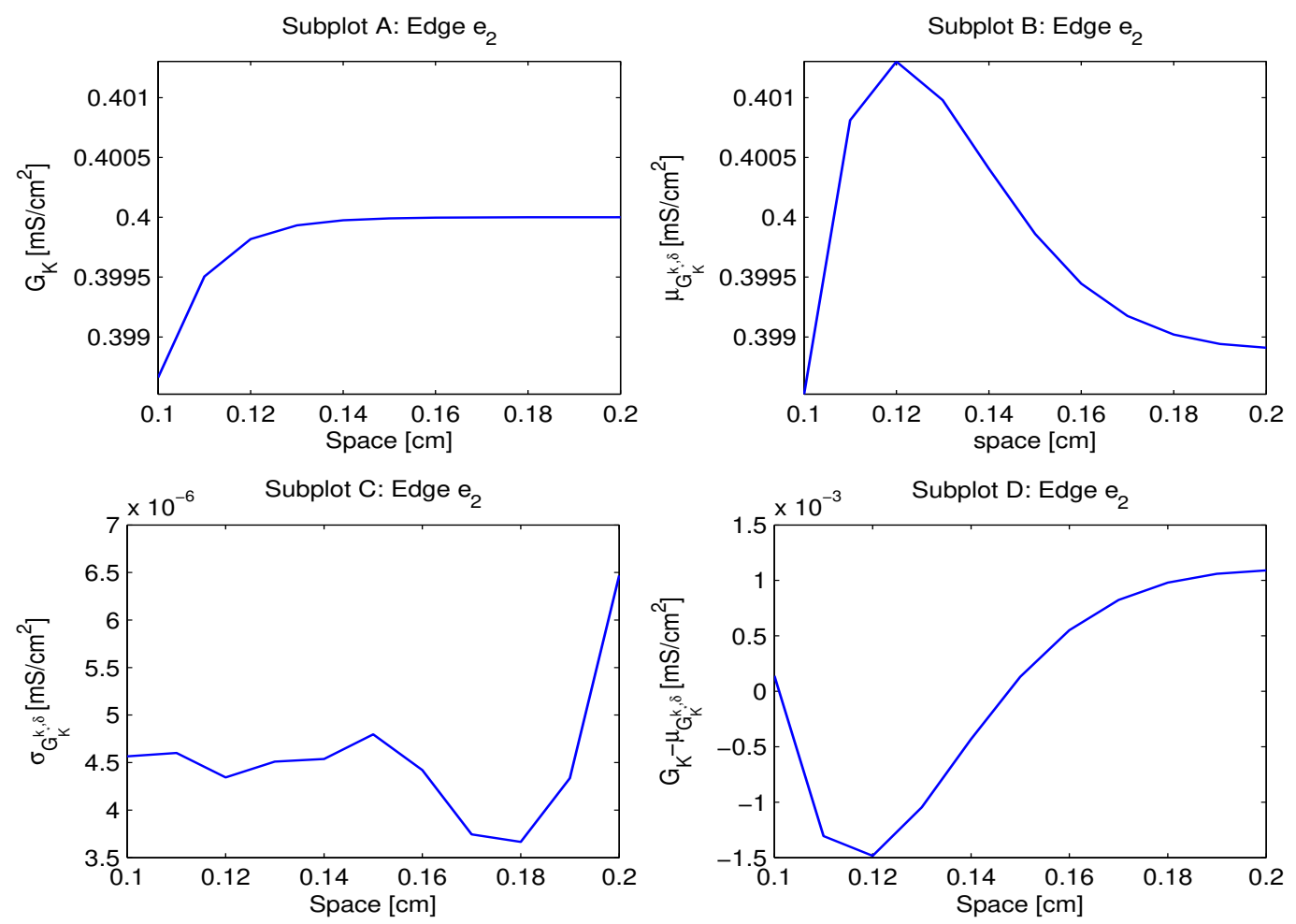

Figure 12. Plots for Example 3.4 and edge $e_{2}$. See Figure 3 for the subplots description.

Integrating by parts twice the first term from Eq. (29) with respect to the space variable, and using the boundary conditions for $W^{k}$ we have

$$
\begin{aligned}
\int_{0}^{L} \int_{0}^{T} \frac{r_{a}}{2 R} U_{x x}^{k}(t, x) W^{k}(t, x) d t d x=\int_{0}^{L} \int_{0}^{T} \frac{r_{a}}{2 R} U^{k}(t, x) W_{x x}^{k}(t, x) d t d x \\
\\
+\left.\frac{r_{a}}{2 R} \int_{0}^{T} U_{x}^{k}(t, x) W^{k}(t, x)\right|_{0} ^{L} d t
\end{aligned}
$$

where we denote $\left.U_{x}^{k}(t, x) W^{k}(t, x)\right|_{0} ^{L}=U^{k}(t, L) W^{k}(t, L)-U^{k}(t, 0) W^{k}(t, 0)$. Similarly, integrating by parts the second term from Eq. (29) with respect to time and using the initial condition of $W^{k}$ and the final condition of $U^{k}$, we gather that

$$
\int_{0}^{L} \int_{0}^{T} C_{M} U_{t}^{k}(t, x) W^{k}(t, x) d t d x=-\int_{0}^{L} \int_{0}^{T} C_{M} U^{k}(t, x) W_{t}^{k}(t, x) d t d x .
$$



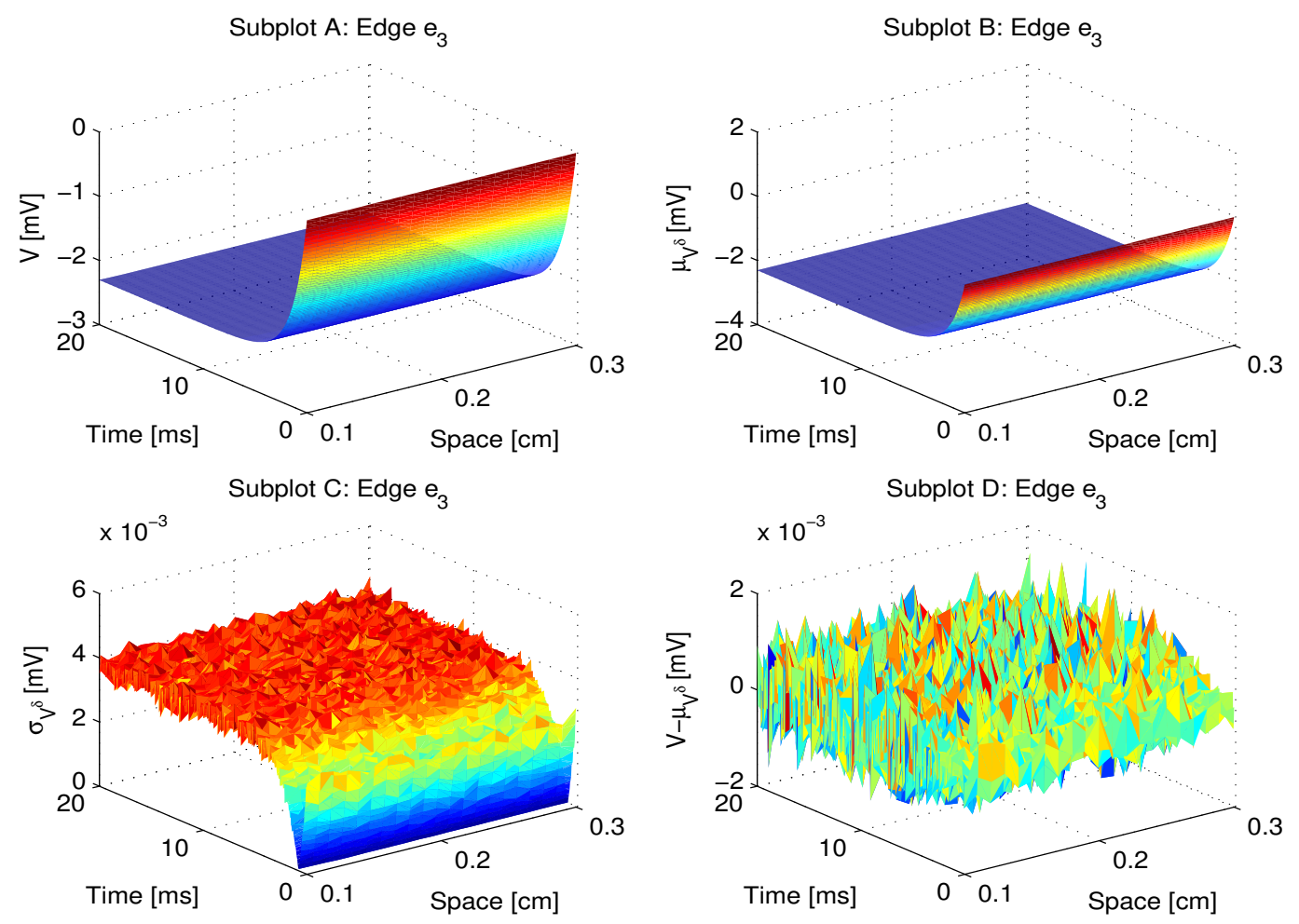

Figure 13. For Example 3.4 and edge $e_{3}$, with $\Delta=1 \%$. See Figure 2 for the subplots description.

Substituting Eqs. 30 and (31) in Eq. 29), it follows that

$$
\begin{gathered}
\int_{0}^{L} \int_{0}^{T}\left(\frac{r_{a}}{2 R} W_{x x}^{k}(t, x)-C_{M} W_{t}^{k}(t, x)-G_{L} W^{k}(t, x)-\sum_{i \in i o n} G_{i}^{k, \delta}(t, x) W^{k}(t, x)\right) U^{k}(t, x) d t d x \\
\quad=-\alpha_{1} \int_{0}^{L} \int_{0}^{T}\left(V^{\delta}(t, x)-V^{k, \delta}(t, x)\right) W^{k}(t, x) d t d x-\left.\frac{r_{a}}{2 R} \int_{0}^{T} U_{x}^{k}(t, x) W^{k}(t, x)\right|_{0} ^{L} d t .
\end{gathered}
$$

Substituting the first equation from 27 in the previous equation, we obtain

$$
\begin{aligned}
& \int_{0}^{L} \int_{0}^{T} \sum_{i \in \operatorname{Ion}} \theta_{i}\left(V^{k, \delta}(t, x)-E_{i}\right) U^{k}(t, x) d t d x \\
& \quad=-\alpha_{1} \int_{0}^{L} \int_{0}^{T}\left(V^{\delta}(t, x)-V^{k, \delta}(t, x)\right) W^{k}(t, x) d t d x-\left.\frac{r_{a}}{2 R} \int_{0}^{T} U_{x}^{k}(t, x) W^{k}(t, x)\right|_{0} ^{L} d t .
\end{aligned}
$$



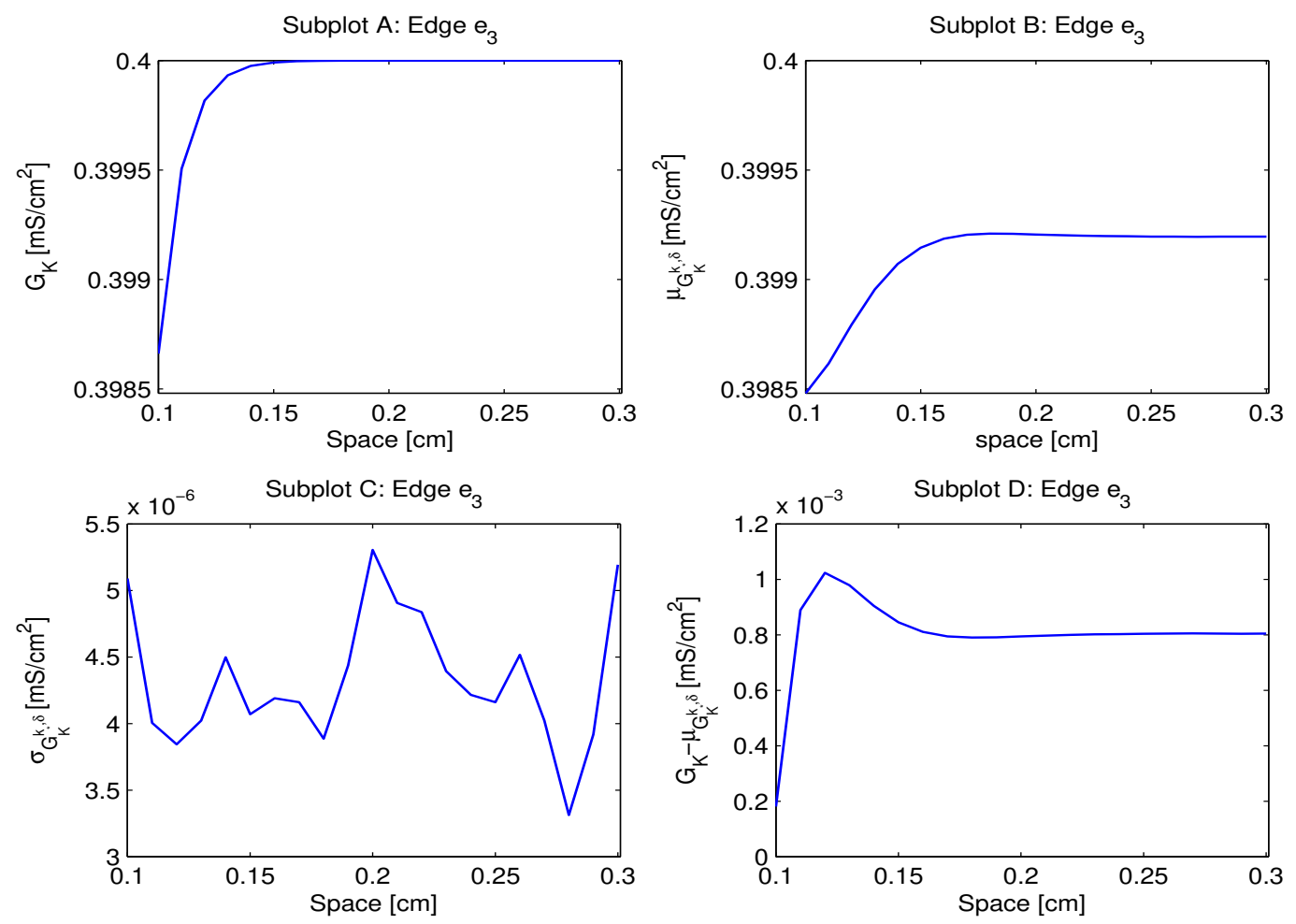

Figure 14. Plots for Example 3.4 and edge $e_{3}$. See Figure 3 for the subplots description.

From the boundary conditions from Eq. (10), the following expression holds:

$$
\begin{aligned}
& \int_{0}^{L} \int_{0}^{T} \sum_{i \in \operatorname{Ion}} \theta_{i}\left(V^{k, \delta}(t, x)-E_{i}\right) U^{k}(t, x) d t d x= \\
&-\alpha_{1} \int_{0}^{L} \int_{0}^{T}\left(V^{\delta}(t, x)-V^{k, \delta}(t, x)\right) W^{k}(t, x) d t d x-\alpha_{2} \int_{0}^{T}\left(V^{\delta}(t, 0)-V^{k, \delta}(t, 0)\right) W^{k}(t, 0) \\
&-\alpha_{2} \int_{0}^{T}\left(V^{\delta}(t, L)-V^{k, \delta}(t, L)\right) W^{k}(t, L) d t .
\end{aligned}
$$

From the previous equation and the definition of the inner product in Eq. (25), we have

$$
\int_{0}^{L} \int_{0}^{T} \sum_{i \in \text { Ion }} \theta_{i}\left(V^{k, \delta}(t, x)-E_{i}\right) U^{k}(t, x) d t d x=-\left\langle\left. V^{\delta}\right|_{\Gamma}-\left.V^{k, \delta}\right|_{\Gamma},\left.W^{k}\right|_{\Gamma}\right\rangle_{R(F)} .
$$

From Eqs. 28) and 32 we have

$$
\begin{aligned}
\int_{0}^{L} \int_{0}^{T} \sum_{i \in \operatorname{Ion}} \theta_{i}\left(G_{i}^{k+1, \delta}(t, x)-G_{i}^{k, \delta}(t, x)\right) d t d x & \\
& =-w^{k, \delta} \int_{0}^{L} \int_{0}^{T} \sum_{i \in \operatorname{Ion}} \theta_{i}\left(V^{k, \delta}(t, x)-E_{i}\right) U^{k}(t, x) d t d x .
\end{aligned}
$$


Since $\boldsymbol{\theta} \in H(F)$ is arbitrary and $L^{\infty}(\Omega)$ is dense in $L^{2}(\Omega)$, we gather that the following iteration holds:

$$
G_{i}^{k+1, \delta}(t, x)=G_{i}^{k, \delta}(t, x)-w^{k, \delta}\left(V^{k, \delta}(t, x)-E_{i}\right) U^{k}(t, x) \quad \text { for all } i \in \text { Ion. }
$$

\section{REFERENCES}

[1] Avdonin, S., And Bell, J. Determining a distributed parameter in a neural cable model via a boundary control method. Journal of mathematical biology 67, 1 (2013), 123-141.

[2] Avdonin, S., And Bell, J. Determining a distributed conductance parameter for a neuronal cable model defined on a tree graph. Journal of Inverse Problems and Imaging 9 (2015), 645-659.

[3] Bédard, C., Béhuret, S., Deleuze, C., Bal, T., and Destexhe, A. Oversampling method to extract excitatory and inhibitory conductances from single-trial membrane potential recordings. Journal of neuroscience methods 210, 1 (2012), 3-14.

[4] BeLL, J. Introduction to theoretical neurobiology. volume 1: Linear cable theory and dendritic structure. volume 2: Nonlinear and stochastic theories (henry c. tuckwell). SIAM Review 32, 1 (1990), 158-160.

[5] Bell, J., And Craciun, G. A distributed parameter identification problem in neuronal cable theory models. Mathematical biosciences 194, 1 (2005), 1-19.

[6] Bezanilla, F. Ion channels: From conductance to structure. Neuron 60, 3 (Nov. 2008), 456-468.

[7] Brown, T. H., Fricke, R. A., and Perkel, D. H. Passive electrical constants in three classes of hippocampal neurons. Journal of Neurophysiology 46, 4 (1981), 812-827.

[8] Casale, A. E., Foust, A. J., Bal, T., And McCormick, D. A. Cortical interneuron subtypes vary in their axonal action potential properties. Journal of Neuroscience 35, 47 (2015), 15555-15567.

[9] Cooley, J., And Dodge Jr, F. Digital computer solutions for excitation and propagation of the nerve impulse. Biophysical journal 6, 5 (1966), 583-599.

[10] Cox, S. J. A new method for extracting cable parameters from input impedance data. Mathematical biosciences 153, 1 (1998), 1-12.

[11] Cox, S. J. An adjoint method for channel localization. Mathematical Medicine and Biology 23, 2 (2006), $139-152$.

[12] Cox, S. J., And Griffith, B. E. Recovering quasi-active properties of dendritic neurons from dual potential recordings. Journal of computational neuroscience 11, 2 (2001), 95-110.

[13] Cox, S. J., AND Ji, L. Identification of the cable parameters in the somatic shunt model. Biological cybernetics 83, 2 (2000), 151-159.

[14] Cox, S. J., AND Ji, L. Discerning ionic currents and their kinetics from input impedance data. Bulletin of mathematical biology 63, 5 (2001), 909-932.

[15] Cox, S. J., AND RAOL, J. H. Recovering the passive properties of tapered dendrites from single and dual potential recordings. Mathematical biosciences 190, 1 (2004), 9-37.

[16] D’Aguanno, A., Bardakjian, B. L., and Carlen, P. L. Passive neuronal membrane parameters: comparison of optimization and peeling methods. IEEE transactions on biomedical engineering 33, 12 (1986), 1188-1196. 
[17] de Myttenaere, A., Golden, B., Grand, B. L., and Rossi, F. Mean absolute percentage error for regression models. Neurocomputing 192 (2016), 38 - 48. Advances in artificial neural networks, machine learning and computational intelligence.

[18] Durand, D., Carlen, P., Gurevich, N., Ho, A., and Kunov, H. Electrotonic parameters of rat dentate granule cells measured using short current pulses and hrp staining. Journal of neurophysiology 50,5 (1983), 1080-1097.

[19] Engl, H. W., Hanke, M., And Neubauer, A. Regularization of inverse problems, vol. 375. Springer Science \& Business Media, 1996.

[20] Ermentrout, G. B., And Terman, D. H. Mathematical foundations of neuroscience, vol. 35. Springer Science \& Business Media, 2010.

[21] George, S., And Sabari, M. Convergence rate results for steepest descent type method for nonlinear ill-posed equations. Applied Mathematics and Computation 294 (2017), 169-179.

[22] Grinvald, A., And Hildesheim, R. Vsdi: a new era in functional imaging of cortical dynamics. Nature Reviews Neuroscience 5 (2004), 874-885.

[23] Hodgkin, A. L., And Huxley, A. F. A quantitative description of membrane current and its application to conduction and excitation in nerve. The Journal of physiology 117, 4 (1952), 500-544.

[24] Holmes, W. R., And Rall, W. Estimating the electrotonic structure of neurons with compartmental models. Journal of Neurophysiology 68, 4 (1992), 1438-1452.

[25] JACK, J., AND REDMAn, S. An electrical description of the motoneurone, and its application to the analysis of synaptic potentials. The Journal of physiology 215, 2 (1971), 321.

[26] Kaltenbacher, B., Neubauer, A., And Scherzer, O. Iterative regularization methods for nonlinear ill-posed problems, vol. 6. Walter de Gruyter, 2008.

[27] Kashef, B., And Bellman, R. Solution of the partial differential equation of the hodgkin-huxley model using differential quadrature. Mathematical Biosciences 19, 1-2 (1974), 1-8.

[28] Kawato, M. Cable properties of a neuron model with non-uniform membrane resistivity. Journal of theoretical biology 111, 1 (1984), 149-169.

[29] KIRsch, A. An introduction to the mathematical theory of inverse problems, vol. 120. Springer Science \& Business Media, 2011.

[30] Kobayashi, R., Shinomoto, S., and Lansky, P. Estimation of time-dependent input from neuronal membrane potential. Neural Computation 23, 12 (2011), 3070-3093.

[31] Kreyszig, E. Introductory functional analysis with applications, vol. 1. wiley New York, 1978.

[32] Mandujano, J. A. V. Examples to estimate conductances: Prgram. avaliable at https://github.com/mandujanovalle/inverse-problem-in-the-cable-equation.

[33] Mascagni, M. The backward euler method for numerical solution of the hodgkin-huxley equations of nerve conduction. SIAM journal on numerical analysis 27, 4 (1990), 941-962.

[34] Mascagni, M. V., Sherman, A. S., ET Al. Numerical methods for neuronal modeling. Methods in neuronal modeling 2 (1989).

[35] NAYAK, A. A new parameter-free regularization method for inverse problems. arXiv e-prints (Mar 2019), arXiv:1903.03130. 
[36] Neubauer, A. A new gradient method for ill-posed problems. Numerical Functional Analysis and Optimization 39, 6 (2018), 737-762.

[37] Pierce, A. Unique identification of eigenvalues and coefficients in a parabolic problem. SIAM Journal on Control and Optimization 17, 4 (1979), 494-499.

[38] RALL, W. Branching dendritic trees and motoneuron membrane resistivity. Experimental neurology 1, 5 (1959), 491-527.

[39] RALL, W. Membrane potential transients and membrane time constant of motoneurons. Experimental neurology 2, 5 (1960), 503-532.

[40] Rall, W. Theory of physiological properties of dendrites. Annals of the New York Academy of Sciences 96, 4 (1962), 1071-1092.

[41] RaLl, W. Core conductor theory and cable properties of neurons. Comprehensive Physiology 1 (1977), 39-97.

[42] Rall, W., Burke, R., Holmes, W., Jack, J., Redman, S., and Segev, I. Matching dendritic neuron models to experimental data. Physiological Reviews 72, 4 (1992), S159-S186.

[43] Schierwagen, A. Identification problems in distributed parameter neuron models. Automatica 26, 4 (1990), 739-755.

[44] Schutter, E. D. Computational modeling methods for neuroscientists. The MIT Press, 2009.

[45] Stuart, G., And Spruston, N. Determinants of voltage attenuation in neocortical pyramidal neuron dendrites. Journal of Neuroscience 18, 10 (1998), 3501-3510.

[46] Tadi, M., Klibanov, M. V., and Cai, W. An inversion method for parabolic equations based on quasireversibility. Computers \&3 Mathematics with Applications 43, 8 (2002), 927-941.

[47] Tuckwell, H. C. Introduction to theoretical neurobiology: volume 2, nonlinear and stochastic theories, vol. 8. Cambridge University Press, 1988.

[48] Vich, C., Berg, R. W., Guillamon, A., and Ditlevsen, S. Estimation of synaptic conductances in presence of nonlinear effects caused by subthreshold ionic currents. Frontiers in computational neuroscience 11 (2017), 69.

[49] Vich, C., and Guillamon, A. Dissecting estimation of conductances in subthreshold regimes. Journal of computational neuroscience 39, 3 (2015), 271-287.

[50] White, J. A., Manis, P. B., and Young, E. D. The parameter identification problem for the somatic shunt model. Biological cybernetics 66, 4 (1992), 307-318.

[51] Willms, A. R., Baro, D. J., Harris-Warrick, R. M., and Guckenheimer, J. An improved parameter estimation method for hodgkin-huxley models. Journal of Computational Neuroscience 6, 2 (1999), 145-168.

[52] Yaşar, T. B., Wright, N. C., And Wessel, R. Inferring presynaptic population spiking from single-trial membrane potential recordings. Journal of neuroscience methods 259 (2016), 13-21.

[53] Zerlaut, Y., and Destexhe, A. Heterogeneous firing responses predict diverse couplings to presynaptic activity in mice layer v pyramidal neurons. PLoS computational biology 13, 4 (2017), e1005452. 
Laboratório Nacional de Computação Científica, Petrópolis - RJ, Brazil

E-mail address: jhimyunac@gmail.com

Laboratório Nacional de Computação Científica, Petrópolis - RJ, Brazil

EPGE - Fundação Getúlio Vargas, Rio de Janeiro - RJ, Brazil

E-mail address: alm@lncc.br, alexandre.madureira@fgv.br

Department of Mathematics, Federal University of St. Catarina, P.O. Box 476, 88040-900 FLORIANÓPOLIS, BRAZIL

E-mail address: acgleitao@gmail.com 